\title{
Solution to the ghost problem in fourth order derivative theories
}

\author{
Philip D. Mannheim* \\ Department of Physics, University of Connecticut, Storrs, CT 06269
}

(Dated: September 6, 2006)

\begin{abstract}
We present a solution to the ghost problem in fourth order derivative theories. In particular we study the Pais-Uhlenbeck fourth order oscillator model, a model which serves as a prototype for theories which are based on second plus fourth order derivative actions. Via a Dirac constraint method quantization we construct the appropriate quantum-mechanical Hamiltonian and Hilbert space for the system. We find that while the second-quantized Fock space of the general PaisUhlenbeck model does indeed contain the negative norm energy eigenstates which are characteristic of higher derivative theories, in the limit in which we switch off the second order action, such ghost states are found to move off shell, with the spectrum of asymptotic in and out S-matrix states of the pure fourth order theory which results being found to be completely devoid of states with either negative energy or negative norm. We confirm these results by quantizing the PaisUhlenbeck theory via path integration and by constructing the associated first-quantized wave mechanics, and show that the disappearance of the would-be ghosts from the energy eigenspectrum in the pure fourth order limit is required by a hidden symmetry that the pure fourth order theory is unexpectedly found to possess. The occurrence of on-shell ghosts is thus seen not to be a shortcoming of pure fourth order theories per se, but rather to be one which only arises when fourth and second order theories are coupled to each other.
\end{abstract}

*Electronic address: philip.mannheim@uconn.edu 


\section{INTRODUCTION}

It is widely believed that theories based on fourth order derivative equations of motion possess unphysical ghost states of negative norm. However, in the literature arguments for so undesirable a state of affairs have actually only been advanced for one particular class of such theories, viz. hybrid ones in which there are both second and fourth order derivatives (see e.g. 1]); and moreover, the discussion has only been formulated within the context of canonical quantization. However, such an analysis is unreliable for two separate reasons. First, higher derivative theories are constrained systems and thus cannot be quantized canonically [2, 3], 4]; and second, the analysis does not address what is to occur in the pure fourth order limit in which the second order piece is switched off. We shall address both of these issues by using the method of Dirac constraints [5] to quantize a prototype second plus fourth order theory, viz. the Pais-Uhlenbeck fourth order oscillator model [6], and on so doing shall discover that the limit in which the second order piece is switched off is a highly singular one in which the would-be ghost states move off the mass shell [7].

To appreciate the singular nature of the switching off of the second order piece, it suffices to consider a classical scalar field theory which is based on the action

$$
I=-\frac{1}{2} \int d^{4} x\left(M^{2} \partial_{\mu} \phi \partial^{\mu} \phi+\partial_{\mu} \partial_{\nu} \phi \partial^{\mu} \partial^{\nu} \phi\right) .
$$

For such an action the equation of motion of the scalar field $\phi$ is given by

$$
\left(-\partial_{t}^{2}+\nabla^{2}\right)\left(-\partial_{t}^{2}+\nabla^{2}-M^{2}\right) \phi(\bar{x}, t)=0
$$

and for this equation of motion one can immediately construct a classical propagator of the form

$$
D^{(4)}\left(k^{2}=-\left(k^{0}\right)^{2}+\bar{k}^{2}, M\right)=\frac{1}{k^{2}\left(k^{2}+M^{2}\right)}=\frac{1}{M^{2} k^{2}}-\frac{1}{M^{2}\left(k^{2}+M^{2}\right)} .
$$

As constructed, the second form given for $D^{(4)}\left(k^{2}, M\right)$ in Eq. (3) contains two pole terms, the first with a positive residue and the second with negative one, to thus suggest that in a quantization of the theory the propagator would then contain two sets of states, one a normal set with positive norm and the second a ghost set with negative norm. However, such an outcome would have no bearing on the status of the pure fourth theory itself, since in the limit in which the parameter $M^{2}$ in Eq. (11) is switched off, the decomposition of $D^{(4)}\left(k^{2}, M\right)$ given as the second form of Eq. (3) becomes undefined. 
To get a sense of what the $M^{2} \rightarrow 0$ limit might in fact look like, it is very instructive to construct the fourth order propagators directly out of the familiar second order retarded and advanced propagators defined as $-(1 / 2 \pi)^{4} \int d^{4} k e^{i k \cdot x} /\left[k^{2}+M^{2} \mp i \epsilon \epsilon\left(k^{0}\right)\right]$, viz.

$$
\begin{aligned}
& D^{(2)}(x, M ; \mathrm{RET})=-\frac{1}{4 \pi r} \delta(t-r)+\frac{M}{4 \pi\left(t^{2}-r^{2}\right)^{1 / 2}} \theta(t-r) J_{1}\left(M\left(t^{2}-r^{2}\right)^{1 / 2}\right) \\
& D^{(2)}(x, M ; \mathrm{ADV})=-\frac{1}{4 \pi r} \delta(t+r)+\frac{M}{4 \pi\left(t^{2}-r^{2}\right)^{1 / 2}} \theta(-t-r) J_{1}\left(M\left(t^{2}-r^{2}\right)^{1 / 2}\right)
\end{aligned}
$$

Specifically, in the same way as the second order propagator equation contains two independent solutions, viz. the retarded and advanced propagators, the fourth order propagator equation should contain four independent solutions. Given the structure of Eq. (3), the four propagators of the fourth order theory can thus be written as linear combinations of the two second order propagators, to thus yield the fourth order retarded, advanced and mixed

$$
\begin{aligned}
D^{(4)}(x, M ; \mathrm{RET}) & =-\frac{1}{M^{2}}\left[D^{(2)}(x, M=0 ; \mathrm{RET})-D^{(2)}(x, M ; \mathrm{RET})\right], \\
D^{(4)}(x, M ; \mathrm{MIXED}) & =-\frac{1}{M^{2}}\left[D^{(2)}(x, M=0 ; \mathrm{RET})-D^{(2)}(x, M ; \mathrm{ADV})\right], \\
D^{(4)}(x, M ; \mathrm{MIXED}) & =-\frac{1}{M^{2}}\left[D^{(2)}(x, M=0 ; \mathrm{ADV})-D^{(2)}(x, M ; \mathrm{RET})\right], \\
D^{(4)}(x, M ; \mathrm{ADV}) & =-\frac{1}{M^{2}}\left[D^{(2)}(x, M=0 ; \mathrm{ADV})-D^{(2)}(x, M ; \mathrm{ADV})\right] .
\end{aligned}
$$

Recalling now that the small argument limit of the $J_{1}(z)$ Bessel function is given as $J_{1}(z) \rightarrow$ $z / 2$, in the limit in which we let $M^{2}$ go to zero, the four propagators of Eq. (5) limit to

$$
\begin{array}{r}
D^{(4)}(x, M=0 ; \mathrm{RET})=\frac{1}{8 \pi} \theta(t-r), \\
D^{(4)}(x, M=0 ; \mathrm{MIXED})=\infty, \\
D^{(4)}(x, M=0 ; \mathrm{MIXED})=\infty, \\
D^{(4)}(x, M=0 ; \mathrm{ADV})=\frac{1}{8 \pi} \theta(-t-r) .
\end{array}
$$

As we see, of the four propagators only two remain finite, the fully retarded and the fully advanced ones, with the two mixed ones becoming undefined. The pure fourth order theory, viz. the one based on the equation of motion $\left(-\partial_{t}^{2}+\nabla^{2}\right)^{2} \phi(\bar{x}, t)=0$ thus only admits of two independent propagators and not four [8], and thus in the quantum theory we can anticipate that there will be only one family of observable states and not two.

To investigate the validity or otherwise of this anticipation, it is convenient to descend from field theory to particle mechanics. We thus restrict to fields which have a spatial 
dependence of plane wave form, and on introducing

$$
\phi(\bar{x}, t)=q(t) e^{i \bar{k} \cdot \bar{x}}, \omega_{1}^{2}+\omega_{2}^{2}=2 \bar{k}^{2}+M^{2} \quad, \quad \omega_{1}^{2} \omega_{2}^{2}=\bar{k}^{4}+\bar{k}^{2} M^{2}
$$

find that $q(t)$ then obeys

$$
\frac{d^{4} q}{d t^{4}}+\left(\omega_{1}^{2}+\omega_{2}^{2}\right) \frac{d^{2} q}{d t^{2}}+\omega_{1}^{2} \omega_{2}^{2} q=0
$$

With this equation of motion being derivable from the acceleration dependent PaisUhlenbeck fourth order oscillator action

$$
I_{\mathrm{PU}}=\int d t L_{\mathrm{PU}}, \quad L_{\mathrm{PU}}=\frac{\gamma}{2}\left[\ddot{q}^{2}-\left(\omega_{1}^{2}+\omega_{2}^{2}\right) \dot{q}^{2}+\omega_{1}^{2} \omega_{2}^{2} q^{2}\right]
$$

where $\gamma$ is a pure constant, we shall thus study the properties of a quantum mechanics based on this $I_{P U}$ in both the unequal and equal frequency limits, with it being the latter equal frequency limit wherein $\omega_{1}^{2}=\omega_{2}^{2}=\bar{k}^{2}$ which corresponds to the switching off of the $M^{2}$ mass

parameter. And as we shall see, this equal frequency limit will prove to be highly singular.

\section{THE CLASSICAL DIRAC CONSTRAINT HAMILTONIAN}

When viewed purely as a classical Lagrangian, the Pais-Uhlenbeck Lagrangian $L_{\mathrm{PU}}$ describes a constrained system since the velocity has to serve as a canonical conjugate to both the position and the acceleration. Thus before one can attempt to quantize the PaisUhlenbeck theory, one must first take care of the constraints already at the classical level by first constructing an appropriate classical Hamiltonian which incorporates them. To do this we follow Dirac and introduce a new variable $x$ which is to serve as the missing canonical variable, and replace $L_{\mathrm{PU}}$ by the Lagrangian

$$
L=\frac{\gamma}{2}\left[\dot{x}^{2}-\left(\omega_{1}^{2}+\omega_{2}^{2}\right) x^{2}+\omega_{1}^{2} \omega_{2}^{2} q^{2}\right]+\lambda(\dot{q}-x)
$$

where $\lambda$ is a Lagrange multiplier. However, since the Lagrange multiplier is introduced to enforce the constraint $\dot{q}=x$ at each time $t, \lambda$ must itself depend on the time $t$, to thus become a yet further dynamical variable. With there thus now being three generalized coordinates, we need to introduce not two but three generalized momenta, viz.

$$
p_{x}=\frac{\partial L}{\partial \dot{x}}=\gamma \dot{x} \quad, \quad p_{q}=\frac{\partial L}{\partial \dot{q}}=\lambda, \quad p_{\lambda}=\frac{\partial L}{\partial \dot{\lambda}}=0
$$


to thereby oblige us to introduce an associated six dynamical variable Hamiltonian $H_{L}$ of the form

$$
H_{L}=p_{q} \dot{q}+p_{x} \dot{x}+p_{\lambda} \dot{\lambda}-\frac{\gamma}{2}\left[\dot{x}^{2}-\left(\omega_{1}^{2}+\omega_{2}^{2}\right) x^{2}+\omega_{1}^{2} \omega_{2}^{2} q^{2}\right]-\lambda(\dot{q}-x)
$$

In order to be able to reduce the theory to just the four dynamical position and momentum variables $q, x, p_{q}$ and $p_{x}$, we need to find a way to make the variables $\lambda$ and $p_{\lambda}$ redundant. Since we could readily do this if we could find a way to implement the conditions $p_{q}-\lambda=0$, $p_{\lambda}=0$ suggested by Eq. (111) as constraints, we introduce two constraint functions

$$
\phi_{1}=p_{q}-\lambda, \phi_{2}=p_{\lambda}
$$

and try to construct a yet further Hamiltonian which would naturally enforce the vanishing of these two constraint functions within an appropriately chosen Poisson bracket algebra [9]. To this end we replace $H_{L}$ by the Hamiltonian

$$
H_{1}=H_{L}+u_{1} \phi_{1}+u_{2} \phi_{2}
$$

viz. by

$$
H_{1}=\frac{p_{x}^{2}}{2 \gamma}+\frac{\gamma}{2}\left(\omega_{1}^{2}+\omega_{2}^{2}\right) x^{2}-\frac{\gamma}{2} \omega_{1}^{2} \omega_{2}^{2} q^{2}+\lambda x+u_{1}\left(p_{q}-\lambda\right)+u_{2} p_{\lambda}
$$

where $u_{1}$ and $u_{2}$ are for the moment arbitrary functions of the coordinates and momenta. With $H_{1}$ depending on six generalized coordinates and momenta, we can define Poisson brackets for this 6-dimensional phase space of the form

$$
\{A, B\}=\frac{\partial A}{\partial x} \frac{\partial B}{\partial p_{x}}-\frac{\partial A}{\partial p_{x}} \frac{\partial B}{\partial x}+\frac{\partial A}{\partial q} \frac{\partial B}{\partial p_{q}}-\frac{\partial A}{\partial p_{q}} \frac{\partial B}{\partial q}+\frac{\partial A}{\partial \lambda} \frac{\partial B}{\partial p_{\lambda}}-\frac{\partial A}{\partial p_{\lambda}} \frac{\partial B}{\partial \lambda} .
$$

With such a definition we immediately obtain a Poisson bracket algebra of the form

$$
\begin{gathered}
\left\{x, p_{x}\right\}=\left\{q, p_{q}\right\}=\left\{\lambda, p_{\lambda}\right\}=1 \\
\left\{\phi_{1}, H_{1}\right\}=\gamma \omega_{1}^{2} \omega_{2}^{2} q-u_{2}+\phi_{1}\left\{\phi_{1}, u_{1}\right\}+\phi_{2}\left\{\phi_{1}, u_{2}\right\} \\
\left\{\phi_{2}, H_{1}\right\}=-x+u_{1}+\phi_{1}\left\{\phi_{2}, u_{1}\right\}+\phi_{2}\left\{\phi_{2}, u_{2}\right\} .
\end{gathered}
$$

Given the Poisson bracket relations of Eq. (18), we thus take the two functions $u_{1}$ and $u_{2}$ to be of the form

$$
u_{1}=x \quad, \quad u_{2}=\gamma \omega_{1}^{2} \omega_{2}^{2} q
$$


with the Hamiltonian then taking the form

$$
H_{2}=\frac{p_{x}^{2}}{2 \gamma}+\frac{\gamma}{2}\left(\omega_{1}^{2}+\omega_{2}^{2}\right) x^{2}-\frac{\gamma}{2} \omega_{1}^{2} \omega_{2}^{2} q^{2}+p_{q} x+\gamma \omega_{1}^{2} \omega_{2}^{2} q p_{\lambda}
$$

and with the constraint functions now being found to obey the Poisson bracket relations

$$
\left\{\phi_{1}, H_{2}\right\}=\gamma \omega_{1}^{2} \omega_{2}^{2} \phi_{2}=\gamma \omega_{1}^{2} \omega_{2}^{2} p_{\lambda} \quad, \quad\left\{\phi_{2}, H_{2}\right\}=\left\{p_{\lambda}, H_{2}\right\}=0
$$

Given Eq. (21), we thus see that within a Poisson bracket algebra defined with respect to the Hamiltonian $H_{2}$, the $\phi_{1}=0, \phi_{2}=0$ constraints can now consistently be imposed. Consequently, if we now introduce a new Hamiltonian

$$
H=\frac{p_{x}^{2}}{2 \gamma}+p_{q} x+\frac{\gamma}{2}\left(\omega_{1}^{2}+\omega_{2}^{2}\right) x^{2}-\frac{\gamma}{2} \omega_{1}^{2} \omega_{2}^{2} q^{2}
$$

and define 4-dimensional phase space Poisson brackets of the form

$$
\{A, B\}=\frac{\partial A}{\partial x} \frac{\partial B}{\partial p_{x}}-\frac{\partial A}{\partial p_{x}} \frac{\partial B}{\partial x}+\frac{\partial A}{\partial q} \frac{\partial B}{\partial p_{q}}-\frac{\partial A}{\partial p_{q}} \frac{\partial B}{\partial q},
$$

we will then obtain a self-contained Poisson bracket algebra of the form

$$
\begin{gathered}
\left\{x, p_{x}\right\}=1,\left\{q, p_{q}\right\}=1,\{x, q\}=\left\{x, p_{q}\right\}=\left\{q, p_{x}\right\}=\left\{p_{x}, p_{q}\right\}=0 \\
\{x, H\}=\frac{p_{x}}{\gamma},\{q, H\}=x,\left\{p_{x}, H\right\}=-p_{q}-\gamma\left(\omega_{1}^{2}+\omega_{2}^{2}\right) x,\left\{p_{q}, H\right\}=\gamma \omega_{1}^{2} \omega_{2}^{2} q .
\end{gathered}
$$

With this algebra being closed, it is thus the one we want, with the now unconstrained four variable Hamiltonian $H$ of Eq. (22) therefore being the appropriate one for the problem. With the Hamiltonian of Eq. (22) being based on two coordinates and two momenta, its phase space thus has the same dimensionality as that of a two-dimensional harmonic oscillator.

\section{STATIONARY SOLUTIONS AND THE OSTROGRADSKI HAMILTONIAN}

Once we have the correct classical Hamiltonian, as well as use it as the generator of Poisson bracket relations, via Hamilton's principle $(\{V, H\}=\dot{V}$ for any dynamical variable $V)$ we can also use it to determine the stationary solutions to the classical equations of motion. Given Eq. (25) these stationary solutions immediately take the form

$$
\dot{x}=\frac{p_{x}}{\gamma} \quad, \quad \dot{q}=x \quad, \quad \dot{p}_{x}=-p_{q}-\gamma\left(\omega_{1}^{2}+\omega_{2}^{2}\right) x \quad, \quad \dot{p}_{q}=\gamma \omega_{1}^{2} \omega_{2}^{2} q
$$


with elimination of $x, p_{x}$ and $p_{q}$ immediately recovering Eq. (8) just as we would want. In this stationary solution we can evaluate the stationary classical Hamiltonian, to find that it takes the form

$$
H_{\mathrm{STAT}}=\frac{\gamma}{2} \ddot{q}^{2}-\frac{\gamma}{2}\left(\omega_{1}^{2}+\omega_{2}^{2}\right) \dot{q}^{2}-\frac{\gamma}{2} \omega_{1}^{2} \omega_{2}^{2} q^{2}-\gamma \dot{q} \frac{d^{3} q}{d t^{3}}
$$

and via use of the equation of motion of Eq. (8) we can readily check that $H_{\text {STAT }}$ is indeed time independent, just as a stationary classical Hamiltonian should be.

As a stationary classical Hamiltonian, the particular $H_{\mathrm{STAT}}$ that we have found can be written in a familiar form. Specifically, it was noted quite some time ago by Ostrogradski 10] that the general Hamiltonian

$$
H_{\mathrm{OST}}=\dot{q} \frac{\partial L}{\partial \dot{q}}+\ddot{q} \frac{\partial L}{\partial \ddot{q}}-\dot{q} \frac{d}{d t}\left(\frac{\partial L}{\partial \ddot{q}}\right)-L
$$

would be a time independent constant of the motion in any solution to the Euler-Lagrange equation of motion

$$
\frac{\partial L}{\partial q}-\frac{d}{d t}\left(\frac{\partial L}{\partial \dot{q}}\right)+\frac{d^{2}}{d t^{2}}\left(\frac{\partial L}{\partial \ddot{q}}\right)=0
$$

which is associated with a Lagrangian which depends on acceleration as well as position and velocity. Direct evaluation of this $H_{\mathrm{OST}}$ for the Pais-Uhlenbeck Lagrangian of interest is immediately found to reveal that $H_{\mathrm{OST}}$ of Eq. (28) and $H_{\mathrm{STAT}}$ of Eq. (27) are nothing less than identical to each other, while direct evaluation of Eq. (29) is found to lead right back to the equation of motion of Eq. (8). With our use of the Dirac constraint method being found to lead us right back to long-established properties of the stationary sector of the classical theory, we are thus assured that the Hamiltonian $H$ of Eq. (22) is indeed the correct Hamiltonian for the problem. And while this Hamiltonian serves purely as an intermediate construct when one is only interested in finding the stationary solutions to the classical theory, since the Poisson bracket algebra of Eqs. (24) and (25) is also defined for nonstationary classical paths as well (i.e. for paths which do not obey Hamilton's equations of motion), and since it is precisely into these non-stationary paths that a quantum-mechanical wave packet can spread [1]], it is thus this selfsame Hamiltonian and Poisson bracket algebra which we can use in order to quantize the theory. However, before actually doing so, it is instructive to first look at the explicit solutions to the classical equations of motion of the Pais-Uhlenbeck theory in a little more detail. 


\section{EXPLICIT STATIONARY CLASSICAL SOLUTIONS}

In the unequal frequency case the general solution to the equation of motion of Eq. (8) contains two oscillators, viz.

$$
q(t)=a_{1} e^{-i \omega_{1} t}+a_{2} e^{-i \omega_{2} t}+\text { c.c. },
$$

with the stationary Hamiltonian being found to evaluate to

$$
H_{\mathrm{STAT}}\left(\omega_{1} \neq \omega_{2}\right)=2 \gamma\left(\omega_{1}^{2}-\omega_{2}^{2}\right)\left(a_{1}^{*} a_{1} \omega_{1}^{2}-a_{2}^{*} a_{2} \omega_{2}^{2}\right)
$$

In the equal frequency case the general solution is of the form

$$
q(t)=c_{1} e^{-i \omega t}+c_{2} t e^{-i \omega t}+\text { c.c. },
$$

with the stationary Hamiltonian

$$
H_{\mathrm{STAT}}\left(\omega_{1}=\omega_{2}=\omega\right)=4 \gamma \omega^{2}\left(2 c_{2}^{*} c_{2}+i \omega c_{1}^{*} c_{2}-i \omega c_{2}^{*} c_{1}\right)
$$

again being time independent despite the presence of the temporal runaway [12].

As regards the structure of the unequal frequency $H_{\mathrm{STAT}}\left(\omega_{1} \neq \omega_{2}\right)$, we see that no matter what the overall sign of the quantity $\gamma\left(\omega_{1}^{2}-\omega_{2}^{2}\right)$, one of its two independent oscillator modes will have to possess negative energy, to thereby make the classical theory unbounded from below. It is this disease which will translate into states of negative norm in the quantized version of the theory. For the equal frequency case however we get a quite different outcome. Specifically, its two independent modes separately have energies

$$
H_{\mathrm{STAT}}\left(\omega_{1}=\omega_{2}=\omega ; c_{2}=0\right)=0
$$

and

$$
H_{\mathrm{STAT}}\left(\omega_{1}=\omega_{2}=\omega ; c_{1}=0\right)=8 \gamma \omega^{2} c_{2}^{*} c_{2}
$$

The pure oscillating $c_{1}$ mode thus has zero energy (there being no $c_{1}^{*} c_{1}$ type term in Eq. (33)), while for an appropriate choice of the sign of the parameter $\gamma$, the temporal runaway solution will actually have positive energy. Thus neither of these two modes has negative energy, and in particular, we see that in and of themselves, temporal runaways need not be associated with problematic negative energies. Comparison of the quite different structures possessed by the unequal and the equal frequency stationary Hamiltonians shows that already in the 
classical theory, wisdom obtained from the unequal frequency theory does not immediately carry over to the equal frequency case, and indeed it could not since the unequal frequency $H_{\mathrm{STAT}}\left(\omega_{1} \neq \omega_{2}\right)$ possesses an overall multiplicative $\omega_{1}^{2}-\omega_{2}^{2}$ factor which actually vanishes in the equal frequency limit. Finally, given the particular energy pattern associated with the equal frequency stationary Hamiltonian which is exhibited in Eqs. (34) and (35), we can anticipate that when quantized the equal frequency theory will not only not possess any energy eigenstates with negative norm, because of the zero energy of the $c_{1}$ mode, its quantum counterpart will not propagate at all, to leave only the quantum counterpart of the runaway $c_{2}$ mode as a perfectly acceptable observable state which has neither negative energy or negative norm.

However, before proceeding to the quantization of the Pais-Uhlenbeck theory, it is of interest to note that the zero energy result of Eq. (34) is reminiscent of the fourth order conformal gravity zero energy theorem of Boulware, Horowitz and Strominger [13] who showed that at the classical level those modes of the conformal gravity theory which were spatially asymptotically flat would possess zero energy. With the exact metric outside of a static, spherically symmetric source in the conformal gravity theory being of the form 14]

$$
d s^{2}=-B(r) d t^{2}+A(r) d r^{2}+r^{2} d \Omega_{2}
$$

where the $B(r)$ and $A(r)$ coefficients are given by

$$
B(r)=\frac{1}{A(r)}=1-\frac{2 \beta}{r}+\gamma r
$$

( $\beta$ and $\gamma$ are constants), we see that the restriction to asymptotic flatness is equivalent to only retaining the standard $1 / r$ Newton potential term in $g_{00}$ while dropping the linearly rising one. Now as such, the temporal runaway of the Pais-Uhlenbeck theory is the temporal analog of the spatially linearly rising potential of the conformal theory [15], and the content of Eq. (34) is that if we drop the solution which is rising linearly in time, the only solution which survives will then have none other than zero energy. With Eq. (34) then we recover an analog of a familiar result. However, of even more significance, with Eq. (35) we see that if we do admit of solutions which are not asymptotically flat, then those solutions can even have positive energy. Thus rather than being a difficulty for fourth order theories, the zero energy theorem of [13] is actually seen to be a plus since it does not preclude the absence of states with positive energy, but rather only those which, as we are now about to show, are not to be associated with observable quantum states at all. 


\section{THE UNEQUAL FREQUENCY QUANTUM-MECHANICAL FOCK SPACE}

Since we have constructed the correct unconstrained Hamiltonian for the classical PaisUhlenbeck theory, to obtain the associated quantum-mechanical theory we can now proceed canonically by replacing the Poisson bracket algebra of Eq. (24) by an analogous quantummechanical commutation algebra of the form

$$
\left[q, p_{q}\right]=\left[x, p_{x}\right]=i, \quad[x, q]=\left[x, p_{q}\right]=\left[q, p_{x}\right]=\left[p_{x}, p_{q}\right]=0
$$

To find a Fock space representation of this algebra we make the identification

$$
\begin{aligned}
& q(t)=a_{1} e^{-i \omega_{1} t}+a_{2} e^{-i \omega_{2} t}+\text { H.c. }, \quad p_{q}(t)=i \gamma \omega_{1} \omega_{2}^{2} a_{1} e^{-i \omega_{1} t}+i \gamma \omega_{1}^{2} \omega_{2} a_{2} e^{-i \omega_{2} t}+\text { H.c. }, \\
& x(t)=-i \omega_{1} a_{1} e^{-i \omega_{1} t}-i \omega_{2} a_{2} e^{-i \omega_{2} t}+\text { H.c. }, \quad p_{x}(t)=-\gamma \omega_{1}^{2} a_{1} e^{-i \omega_{1} t}-\gamma \omega_{2}^{2} a_{2} e^{-i \omega_{2} t}+\text { H.c. },
\end{aligned}
$$

to find that the creation and annihilation operators have to obey a commutation algebra of the form

$$
\left[a_{1}, a_{1}^{\dagger}\right]=\frac{1}{2 \gamma \omega_{1}\left(\omega_{1}^{2}-\omega_{2}^{2}\right)}, \quad\left[a_{2}, a_{2}^{\dagger}\right]=\frac{1}{\left.2 \gamma \omega_{2}\left(\omega_{2}^{2}-\omega_{1}^{2}\right)\right]}, \quad\left[a_{1}, a_{2}^{\dagger}\right]=0 \quad, \quad\left[a_{1}, a_{2}\right]=0
$$

On taking all of $\omega_{1}, \omega_{2}$ and $\gamma\left(\omega_{1}^{2}-\omega_{2}^{2}\right)$ to be positive (for definitiveness), we see that while the $\left[a_{1}, a_{1}^{\dagger}\right]$ commutator has the conventional positive sign, the $\left[a_{2}, a_{2}^{\dagger}\right]$ commutator is negative, with there thus being ghost states in this latter sector.

To identify the energy spectrum of the theory we write the quantum-mechanical Hamiltonian in the Fock space basis by inserting Eq. (39) into Eq. (22), to find that the Hamiltonian then takes the form

$$
H=2 \gamma\left(\omega_{1}^{2}-\omega_{2}^{2}\right)\left(\omega_{1}^{2} a_{1}^{\dagger} a_{1}-\omega_{2}^{2} a_{2}^{\dagger} a_{2}\right)+\frac{1}{2}\left(\omega_{1}+\omega_{2}\right)
$$

a form which we immediately recognize as being the quantum-mechanical counterpart of $H_{\mathrm{STAT}}\left(\omega_{1} \neq \omega_{2}\right)$ of Eq. (31) just as one would want. On defining the Fock vacuum according to

$$
a_{1}|\Omega\rangle=0, a_{2}|\Omega\rangle=0,
$$

we then find that the one-particle states

$$
|+1\rangle=\left[2 \gamma \omega_{1}\left(\omega_{1}^{2}-\omega_{2}^{2}\right)\right]^{1 / 2} a_{1}^{\dagger}|\Omega\rangle, \quad|-1\rangle=\left[2 \gamma \omega_{2}\left(\omega_{1}^{2}-\omega_{2}^{2}\right)\right]^{1 / 2} a_{2}^{\dagger}|\Omega\rangle
$$


are both positive energy eigenstates of the Hamiltonian of Eq. (41) with respective energies $\omega_{1}$ and $\omega_{2}$ above the ground state. With the state $|+1\rangle$ being of positive norm and the state $|-1\rangle$ being of negative norm, we conclude that the unequal frequency quantummechanical Pais-Uhlenbeck theory possesses energy eigenstates of negative norm, to thus threaten the physical viability of the theory [16]. However, noting that the commutation relations of Eq. (40) become singular in the equal frequency limit while the Hamiltonian of Eq. (41) develops a zero, the wisdom obtained from the unequal frequency Fock space structure cannot be transported to the equal frequency case. Rather, its Fock space has to be constructed all over again.

\section{THE EQUAL FREQUENCY QUANTUM-MECHANICAL FOCK SPACE}

To construct the equal frequency Fock space we first need to reformulate the unequal frequency Fock space in a new creation and annihilation operator basis whose equal-frequency limit will not in fact be singular. To this end we introduce new operators

$$
\begin{aligned}
& a_{1}=\frac{1}{2}\left(a-b+\frac{2 b \omega}{\epsilon}\right), \quad a_{2}=\frac{1}{2}\left(a-b-\frac{2 b \omega}{\epsilon}\right), \\
& a=a_{1}\left(1+\frac{\epsilon}{2 \omega}\right)+a_{2}\left(1-\frac{\epsilon}{2 \omega}\right), \quad b=\frac{\epsilon}{2 \omega}\left(a_{1}-a_{2}\right),
\end{aligned}
$$

where we have set

$$
\omega=\frac{\left(\omega_{1}+\omega_{2}\right)}{2}, \epsilon=\frac{\left(\omega_{1}-\omega_{2}\right)}{2}
$$

These new variables obey the commutation algebra

$$
\left[a, a^{\dagger}\right]=\lambda, \quad\left[a, b^{\dagger}\right]=\mu \quad, \quad\left[b, a^{\dagger}\right]=\mu \quad, \quad\left[b, b^{\dagger}\right]=\nu \quad, \quad[a, b]=0,
$$

where

$$
\lambda=\nu=-\frac{\epsilon^{2}}{16 \gamma\left(\omega^{2}-\epsilon^{2}\right) \omega^{3}} \quad, \quad \mu=\frac{\left(2 \omega^{2}-\epsilon^{2}\right)}{16 \gamma\left(\omega^{2}-\epsilon^{2}\right) \omega^{3}},
$$

with this commutation algebra indeed being well-behaved in the $\epsilon \rightarrow 0$ limit. In terms of these new operators the position operator takes the form

$$
q(\epsilon \neq 0)=e^{-i \omega t}\left[(a-b) \cos \epsilon t-\frac{2 i b \omega}{\epsilon} \sin \epsilon t\right]+\text { H.c. },
$$

while the Hamiltonian of Eq. (41) is rewritten as

$$
H(\epsilon \neq 0)=8 \gamma \omega^{2} \epsilon^{2}\left(a^{\dagger} a-b^{\dagger} b\right)+8 \gamma \omega^{4}\left(2 b^{\dagger} b+a^{\dagger} b+b^{\dagger} a\right)+\omega
$$


From the structure of the commutation relations of Eq. (46) we find that with respect to the vacuum $|\Omega\rangle$ that both $a$ and $b$ annihilate, prior to setting $\epsilon$ equal to zero neither of the states $a^{\dagger}|\Omega\rangle$ or $b^{\dagger}|\Omega\rangle$ is an eigenstate of $H(\epsilon \neq 0)$. Rather the action of the unequal frequency Hamiltonian on them yields

$$
\begin{aligned}
& H(\epsilon \neq 0) a^{\dagger}|\Omega\rangle=\frac{1}{2 \omega}\left[\left(4 \omega^{2}+\epsilon^{2}\right) a^{\dagger}|\Omega\rangle+\left(4 \omega^{2}-\epsilon^{2}\right) b^{\dagger}|\Omega\rangle\right], \\
& H(\epsilon \neq 0) b^{\dagger}|\Omega\rangle=\frac{1}{2 \omega}\left[\epsilon^{2} a^{\dagger}|\Omega\rangle+\left(4 \omega^{2}-\epsilon^{2}\right) b^{\dagger}|\Omega\rangle\right]
\end{aligned}
$$

with the Hamiltonian acting in the one-particle sector as the non-diagonal matrix

$$
M(\epsilon \neq 0)=\frac{1}{2 \omega}\left(\begin{array}{cc}
4 \omega^{2}+\epsilon^{2} & 4 \omega^{2}-\epsilon^{2} \\
\epsilon^{2} & 4 \omega^{2}-\epsilon^{2}
\end{array}\right) .
$$

Diagonalization of $M(\epsilon \neq 0)$ is straightforward, with the one-particle sector being found to diagonalize the Hamiltonian according to the two states

$$
H(\epsilon \neq 0)|2 \omega \pm \epsilon\rangle=(2 \omega \pm \epsilon)|2 \omega \pm \epsilon\rangle
$$

where

$$
|2 \omega \pm \epsilon\rangle=\left[ \pm \epsilon a^{\dagger}+(2 \omega \mp \epsilon) b^{\dagger}\right]|\Omega\rangle
$$

These states are of course the previous $a_{1}^{\dagger}|\Omega\rangle$ and $a_{2}^{\dagger}|\Omega\rangle$ energy eigenstates states as transcribed to the new basis. However, what will be of far more significance for the $|2 \omega \pm \epsilon\rangle$ states in the following is that in the $\epsilon \rightarrow 0$ limit, they both collapse into one and the same single state, viz. the state $b^{\dagger}|\Omega\rangle$.

Despite the fact that the $a^{\dagger}|\Omega\rangle, b^{\dagger}|\Omega\rangle$ basis is not the one which diagonalizes the $\epsilon \neq 0$ Hamiltonian, the great utility of this particular basis is that in it both $q(\epsilon \neq 0)$ and $H(\epsilon \neq 0)$ (and likewise $p_{q}(\epsilon \neq 0), x(\epsilon \neq 0)$ and $\left.p_{x}(\epsilon \neq 0)\right)$ have well-behaved $\epsilon \rightarrow 0$ limits, viz.

$$
q(\epsilon=0)=e^{-i \omega t}(a-b-2 i b \omega t)+\text { H.c. },
$$

and

$$
H(\epsilon=0)=8 \gamma \omega^{4}\left(2 b^{\dagger} b+a^{\dagger} b+b^{\dagger} a\right)+\omega
$$

We immediately recognize Eqs. (154) and (155) as being the quantum-mechanical counterparts of Eqs. (32) and (33) just as we would want, to thus confirm that the $a^{\dagger}|\Omega\rangle, b^{\dagger}|\Omega\rangle$ basis is indeed the appropriate one for the $\epsilon=0$ theory. In the $\epsilon=0$ limit we find that various 
commutators of interest take the form

$$
\begin{aligned}
& {\left[H(\epsilon=0), a^{\dagger}\right]=\omega\left(a^{\dagger}+2 b^{\dagger}\right),[H(\epsilon=0), a]=-\omega(a+2 b),} \\
& {\left[H(\epsilon=0), b^{\dagger}\right]=\omega b^{\dagger},[H(\epsilon=0), b]=-\omega b,} \\
& {\left[a+b, a^{\dagger}+b^{\dagger}\right]=2 \mu(\epsilon=0),\left[a-b, a^{\dagger}-b^{\dagger}\right]=-2 \mu(\epsilon=0),\left[a+b, a^{\dagger}-b^{\dagger}\right]=0,}
\end{aligned}
$$

with the action of $H(\epsilon=0)$ on the one-particle sector being of the form

$$
\begin{aligned}
& H(\epsilon=0) a^{\dagger}|\Omega\rangle=2 \omega\left[a^{\dagger}|\Omega\rangle+b^{\dagger}|\Omega\rangle\right] \\
& H(\epsilon=0) b^{\dagger}|\Omega\rangle=2 \omega b^{\dagger}|\Omega\rangle
\end{aligned}
$$

As we see from the form of Eq. (57) and equally from the structure of Eqs. (52) and (53), $H(\epsilon=0)$ possesses just one one-particle state, viz. the state $b^{\dagger}|\Omega\rangle$, and not two, with the state $a^{\dagger}|\Omega\rangle$ having moved off shell. With the norm of $b^{\dagger}|\Omega\rangle$ not being negative in the $\epsilon=0$ limit $\left(\left\langle\Omega\left|b b^{\dagger}\right| \Omega\right\rangle=0\right.$ is actually zero - a peculiar but nonetheless perfectly acceptable outcome which we shall address further below), and with its energy being positive, and with this selfsame pattern being found to repeat for the multiparticle states as well, we find

that the quantum-mechanical equal frequency Pais-Uhlenbeck theory possesses no states of negative norm or negative energy whatsoever [17]. Thus reminiscent of the way quantization stabilizes the classical hydrogen atom, once again we see that it is quantum mechanics which serves to render a theory, this time the fourth order Pais-Uhlenbeck theory, viable.

\section{WHY THE EQUAL FREQUENCY THEORY IS VIABLE}

While we have been able to show that the equal frequency Pais-Uhlenbeck theory is physically viable, it is at first perplexing that the equal frequency Hamiltonian $H(\epsilon=0)$ would possess fewer eigenstates than the full Fock space in which the operators $q(\epsilon=0)$, $p_{q}(\epsilon=0), x(\epsilon=0)$ and $p_{x}(\epsilon=0)$ act, with the dimensionality of the spectrum of onshell states of the $H(\epsilon=0)$ Hamiltonian being the same as that of a one-dimensional oscillator rather than that of the two-dimensional one which is associated with the unequal frequency Hamiltonian $H(\epsilon \neq 0)$. Additionally, it is somewhat puzzling that the energy eigenstates that $H(\epsilon=0)$ does actually possess have eigenvalues which are real since the $\epsilon \rightarrow 0$ limit of the matrix $M(\epsilon=0)$ of Eq. (51) is not Hermitian. While there is no theorem which would forbid a non-Hermitian matrix from having real eigenvalues (being Hermitian 
is only sufficient to secure the reality of eigenvalues but not necessary), nonetheless if a non-Hermitian matrix is to have real eigenvalues, some justification is required.

To address both of these issues we need to reconsider the $\epsilon \neq 0$ theory. When written in the $a_{1}^{\dagger}|\Omega\rangle, a_{2}^{\dagger}|\Omega\rangle$ basis the one-particle sector of the $\epsilon \neq 0$ Hamiltonian $H$ of Eq. (41) is manifestly Hermitian with manifestly real eigenvalues. With the transformation of Eq. (44) to the $a^{\dagger}|\Omega\rangle, b^{\dagger}|\Omega\rangle$ basis being a similarity transformation, the eigenvalues have to be left untouched, with the eigenvalues thus remaining real. However, the transformation of Eq. (44) is not an orthogonal one. Rather, it is a transformation to skew axes which acts as

$$
S\left(\begin{array}{cc}
2 \omega+\epsilon & 0 \\
0 & 2 \omega-\epsilon
\end{array}\right) S^{-1}=\frac{1}{2 \omega}\left(\begin{array}{cc}
4 \omega^{2}+\epsilon^{2} & 4 \omega^{2}-\epsilon^{2} \\
\epsilon^{2} & 4 \omega^{2}-\epsilon^{2}
\end{array}\right)=M(\epsilon \neq 0)
$$

where

$$
\begin{aligned}
S & =\frac{1}{2 \epsilon \omega^{1 / 2}(2 \omega+\epsilon)^{1 / 2}}\left(\begin{array}{cc}
2 \omega+\epsilon & -\left(4 \omega^{2}-\epsilon^{2}\right) \epsilon \\
\epsilon & (2 \omega+\epsilon) \epsilon^{2}
\end{array}\right), \\
S^{-1} & =\frac{1}{2 \epsilon \omega^{1 / 2}(2 \omega+\epsilon)^{1 / 2}}\left(\begin{array}{cc}
(2 \omega+\epsilon) \epsilon^{2} & \left(4 \omega^{2}-\epsilon^{2}\right) \epsilon \\
-\epsilon & 2 \omega+\epsilon
\end{array}\right),
\end{aligned}
$$

to thus yield a matrix $M(\epsilon \neq 0)$ which is not Hermitian with respect to the skew basis. The class of matrices which are guaranteed to have real eigenvalues thus includes not just Hermitian matrices themselves but also any non-Hermitian matrices which can be reached from Hermitian matrices via similarity transformations to skew axes. Then, with the $\epsilon \rightarrow 0$ limit of the matrix $M(\epsilon \neq 0)$ of Eq. (58) not being singular, we see that in the limit the matrix $M(\epsilon=0)$ will take the form

$$
M(\epsilon=0)=2 \omega\left(\begin{array}{ll}
1 & 1 \\
0 & 1
\end{array}\right)=2 \omega\left(\begin{array}{ll}
1 & 0 \\
0 & 1
\end{array}\right)+2 \omega\left(\begin{array}{ll}
0 & 1 \\
0 & 0
\end{array}\right),
$$

and still have real eigenvalues (both of which are equal to $2 \omega$ ) even though it remains non-Hermitian.

Now while the above discussion explains why the eigenvalues of the matrix $M(\epsilon=0)$ will all be real, it does not yet explain what happened to the second eigenstate which had been present when $\epsilon$ was non-zero. With both eigenvalues of $M(\epsilon=0)$ being equal to $2 \omega$, we can set up an eigenvalue problem for them, to find that the associated eigenvectors have to obey

$$
\left(\begin{array}{ll}
1 & 1 \\
0 & 1
\end{array}\right)\left(\begin{array}{l}
p \\
q
\end{array}\right)=\left(\begin{array}{c}
p+q \\
q
\end{array}\right)=\left(\begin{array}{l}
p \\
q
\end{array}\right)
$$


a relation for which there is only one solution, viz. $q=0$. As such then, the $M(\epsilon=0)$ matrix only possesses one eigenvector (recall the $\epsilon \rightarrow 0$ behavior of Eq. (53)), with the number of its eigenvectors being less than the number of its eigenvalues. Matrices which possess this property are said to be defective, with these matrices acting on spaces which are not complete. Now in matrix theory Jordan showed that an arbitrary matrix can either be diagonalized by a similarity transformation or brought to the so-called Jordan block form in which all of its non-zero elements can be restricted to being on the diagonal itself and on just one of its sides, with there being no non-zero elements at all on the other (c.f. the first form in Eq. (60) ). Such Jordan block matrices have the same number of eigenvalues as the dimensionality of the matrix, and even have all the eigenvalues already on the diagonal (the zeroes on one side of the diagonal prevent any non-zero elements on the other side from contributing to the secular equation), but because they are in the form of a sum of two matrices one of which is diagonal and the other a divisor of zero (c.f. the second form in Eq. (60) $)$, they do not have a full complement of eigenvectors. With the $M(\epsilon=0)$ matrix being of the Jordan block form it is defective and thus lacks an eigenvector [18].

Now while the $\epsilon \neq 0$ matrix $M(\epsilon \neq 0)$ of Eq. (151) is non-Hermitian, it itself is not a Jordan block matrix. Rather, it possesses two independent eigenvalues and two independent eigenvectors as exhibited in Eq. (53), as it indeed must since $H(\epsilon \neq 0)$ of Eq. (49) is reachable from the $\epsilon \neq 0 \mathrm{H}$ of Eq. (41) (a Hamiltonian whose one-particle sector is Hermitian) by the similarity transformation given in Eq. (59). It is only in the limit in which we set $\epsilon$ to zero that the $M(\epsilon=0)$ matrix goes into the Jordan block form and becomes defective. The $\epsilon \rightarrow 0$ limit is thus a singular one in which one can no longer get back to a diagonal Hamiltonian, with the similarity transformation of Eq. (59) becoming singular (in Eq. (44) both $a_{1}$ and $a_{2}$ possess terms which behave as $\left.1 / \epsilon\right)$. The essence of the solution to the ghost problem in the Pais-Uhlenbeck theory then is that the relevant $\epsilon \neq 0$ and $\epsilon=0$ bases are connected by a similarity transformation which becomes singular in the $\epsilon \rightarrow 0$ limit, with these bases then becoming disconnected, and with the ghosts no longer being able to appear on shell as asymptotic in and out states of the equal frequency theory S-matrix [19].

In our construction of the on-shell Hilbert space of the $H(\epsilon=0)$ Hamiltonian, we only needed to construct its eigenstates, viz. those ket vectors which obey $H(\epsilon=0)|\psi\rangle=E|\psi\rangle$. As such, this eigenvalue problem makes no reference to the space of the dual vectors, and there is thus some freedom in choosing the appropriate bra vectors [4, 20]. (That there is 
this freedom is because in the eigenvalue equation the Hamiltonian acts linearly on the wave function while the norm $\langle\psi \mid \psi\rangle$ is bilinear, with it thus being of no relevance to the linear equation what bilinear norm one might choose to consider.) Thus rather than simply take the dual vectors to be the conjugates of the ket vectors so that the norms of the states $a^{\dagger}|\Omega\rangle$, $b^{\dagger}|\Omega\rangle$ are then given by $\left\langle\Omega\left|a a^{\dagger}\right| \Omega\right\rangle$ and $\left\langle\Omega\left|b b^{\dagger}\right| \Omega\right\rangle$ (both of which norms are actually zero in the equal frequency limit), we can avoid the presence of such zero norm states by instead considering an alternate choice for the dual vectors. With respect to an appropriately chosen matrix $C$ we thus define the dual vectors to be the $C$-conjugates of the kets and introduce a $C$-norm $\langle\psi|C| \psi\rangle$. Thus, if we explicitly take $C$ to act as the Pauli matrix $\sigma_{1}$ in the $a^{\dagger}|\Omega\rangle$, $b^{\dagger}|\Omega\rangle$ space, so that

$$
C a^{\dagger}|\Omega\rangle=b^{\dagger}|\Omega\rangle, \quad C b^{\dagger}|\Omega\rangle=a^{\dagger}|\Omega\rangle,
$$

we then find that one-particle sector matrix elements are of the form

$$
\begin{gathered}
\left\langle\Omega\left|a C a^{\dagger}\right| \Omega\right\rangle=\frac{1}{8 \gamma \omega^{3}}, \quad\left\langle\Omega\left|a C b^{\dagger}\right| \Omega\right\rangle=0, \\
\left\langle\Omega\left|b C a^{\dagger}\right| \Omega\right\rangle=0, \quad\left\langle\Omega\left|b C b^{\dagger}\right| \Omega\right\rangle=\frac{1}{8 \gamma \omega^{3}},
\end{gathered}
$$

with the $a^{\dagger}|\Omega\rangle$ and $b^{\dagger}|\Omega\rangle$ states now forming an orthonormalizable pair of one-particle states, and with the $b^{\dagger}|\Omega\rangle$ state now being an energy eigenstate with a completely acceptable positive norm [21].

As well as analyze the Pais-Uhlenbeck theory from the perspective of its second-quantized Fock space formulation, it is also of interest to analyze the theory from the perspectives of path integral quantization and first-quantized wave mechanics. This we do in appendices A and $\mathrm{B}$, and as we shall see, it will provide additional insight into and confirmation of the structure of the fourth order theory that we have presented here. Additionally, in appendix $\mathrm{C}$ we provide further insight into the defective nature of the equal frequency Hamiltonian by showing that in the equal frequency limit, the Hamiltonian acquires a hidden symmetry which sharply curtails the number of energy eigenstates.

\section{Acknowledgments}

The author wishes to thank Dr. A. Davidson for his active participation in many aspects of this work, and especially in the development of the material presented in appendix C. The author would also like to thank Dr. A. Kovner for some very helpful comments. 


\section{APPENDIX A: PATH INTEGRATION FOR THE FOURTH ORDER THEORY}

To determine the measure of paths needed for path integral quantization, our strategy is to use the selfsame set of paths which are needed for the underlying classical variation problem, and so we shall first determine the appropriate set of variational paths explicitly. There are actually two cases to consider, a coordinate space Euler-Lagrange variation of the $I_{\mathrm{PU}}$ action of Eq. (9), viz.

$$
I_{\mathrm{PU}}=\frac{\gamma}{2} \int_{t_{i}}^{t_{f}} d t\left[\ddot{q}^{2}-\left(\omega_{1}^{2}+\omega_{2}^{2}\right) \dot{q}^{2}+\omega_{1}^{2} \omega_{2}^{2} q^{2}\right],
$$

and a phase space Hamilton variation of the action associated with the Hamiltonian of Eq. (22), viz.

$$
I_{\mathrm{HAM}}=\int_{t_{i}}^{t_{f}} d t\left[p_{q} \dot{q}+p_{x} \dot{x}-\frac{p_{x}^{2}}{2 \gamma}-p_{q} x-\frac{\gamma}{2}\left(\omega_{1}^{2}+\omega_{2}^{2}\right) x^{2}+\frac{\gamma}{2} \omega_{1}^{2} \omega_{2}^{2} q^{2}\right] .
$$

For the coordinate space case first, if we replace $q$ by $q+\delta q$ in $I_{\mathrm{PU}}$, in first order in the variation we will obtain terms which depend on $\delta q, \delta \dot{q}$ and $\delta \ddot{q}$. To bring all of these terms to a pure $\delta q$ form we will need to integrate by parts, and will thus generate surface terms which depend on $\delta q$ and $\delta \dot{q}$. Since functional variation requires that end points be held fixed, we thus vary the action over all paths which have common end-point values of $q$ and $\dot{q}$. The classical Euler-Lagrange functional variation thus has to be made over paths which have a specified $q\left(t_{i}\right)$ and $\dot{q}\left(t_{i}\right)$ at the initial time $t_{i}$, and a specified $q\left(t_{f}\right)$ and $\dot{q}\left(t_{f}\right)$ at the final time

$t_{f}$. That we would need to provide four pieces of information in a fourth order theory is not the key point here - rather it is that those pieces of information must consist of initial and final positions and velocities, and not, say, initial and final positions and accelerations. (This same conclusion was also reached by Hawking and Hertog [3] by consideration not of the classical theory but of the structure of the quantum-mechanical path integral itself.)

To specify a basis of paths we introduce the stationary classical path between fixed end points as given in Eq. (30), viz.

$$
q_{\mathrm{STAT}}(t)=a_{1} e^{-i \omega_{1} t}+a_{2} e^{-i \omega_{2} t}+a_{1}^{*} e^{i \omega_{1} t}+a_{2}^{*} e^{i \omega_{2} t}
$$

[with the real and imaginary parts of $a_{1}$ and $a_{2}$ being expressible in terms of $q\left(t_{i}\right), \dot{q}\left(t_{i}\right)$, $q\left(t_{f}\right)$ and $\left.\dot{q}\left(t_{f}\right)\right]$, and then take the arbitrary path to be of the form

$$
q(t)=q_{\mathrm{STAT}}(t)+\sum_{n=1}^{\infty} a_{n}\left[\cos \left(\frac{n \pi t}{T}\right)-1\right]
$$


where the $a_{n}$ are constants which are constrained according to

$$
\sum_{n=1}^{\infty} a_{n}\left[(-1)^{n}-1\right]=-2 \sum_{n=\text { odd }}^{\infty} a_{n}=0
$$

and where we have set $t_{i}=0$ and $t_{f}=T$. With the arbitrary velocity and acceleration then being given by

$$
\begin{aligned}
& \dot{q}(t)=\dot{q}_{\mathrm{STAT}}(t)-\sum_{n=1}^{\infty} a_{n} \frac{n \pi}{T} \sin \left(\frac{n \pi t}{T}\right), \\
& \ddot{q}(t)=\ddot{q}_{\mathrm{STAT}}(t)-\sum_{n=1}^{\infty} a_{n} \frac{n^{2} \pi^{2}}{T^{2}} \cos \left(\frac{n \pi t}{T}\right),
\end{aligned}
$$

the constraint of Eq. (A5) ensures that in the arbitrary path the general position and general velocity are indeed respectively equal to $q_{\mathrm{STAT}}$ and $\dot{q}_{\mathrm{STAT}}(t)$ at both end points, with the general acceleration not being constrained to be equal to $\ddot{q}_{\mathrm{STAT}}(t)$ at the end points, just as required. In terms of the stationary classical action

$$
\begin{aligned}
I_{\mathrm{STAT}}\left(a_{1}, a_{2}\right) & =\frac{\gamma}{2} \int_{0}^{T} d t\left[\ddot{q}_{\mathrm{STAT}}^{2}-\left(\omega_{1}^{2}+\omega_{2}^{2}\right) \dot{q}_{\mathrm{STAT}}^{2}+\omega_{1}^{2} \omega_{2}^{2} q_{\mathrm{STAT}}^{2}\right] \\
& =\gamma\left(\omega_{1}^{2}+\omega_{2}^{2}\right) \int_{0}^{T} d t\left[\omega_{1}^{2}\left(a_{1}^{2} e^{-2 i \omega_{1} t}+a_{1}^{* 2} e^{2 i \omega_{1} t}\right)+\omega_{2}^{2}\left(a_{2}^{2} e^{-2 i \omega_{2} t}+a_{2}^{* 2} e^{2 i \omega_{2} t}\right)\right] \\
& +\gamma \omega_{1} \omega_{2}\left(\omega_{1}+\omega_{2}\right)^{2} \int_{0}^{T} d t\left[a_{1} a_{2} e^{-i\left(\omega_{1}+\omega_{2}\right) t}+a_{1}^{*} a_{2}^{*} e^{i\left(\omega_{1}+\omega_{2}\right) t}\right] \\
& -\gamma \omega_{1} \omega_{2}\left(\omega_{1}-\omega_{2}\right)^{2} \int_{0}^{T} d t\left[a_{1} a_{2}^{*} e^{-i\left(\omega_{1}-\omega_{2}\right) t}+a_{1}^{*} a_{2} e^{i\left(\omega_{1}-\omega_{2}\right) t}\right] \\
& =\frac{i \gamma\left(\omega_{1}^{2}+\omega_{2}^{2}\right)}{2}\left[\omega_{1} a_{1}^{2}\left(e^{-2 i \omega_{1} T}-1\right)-\omega_{1} a_{1}^{* 2}\left(e^{2 i \omega_{1} T}-1\right)\right] \\
& +\frac{i \gamma\left(\omega_{1}^{2}+\omega_{2}^{2}\right)}{2}\left[\omega_{2} a_{2}^{2}\left(e^{-2 i \omega_{2} T}-1\right)-\omega_{2} a_{2}^{* 2}\left(e^{2 i \omega_{2} T}-1\right)\right] \\
& +i \gamma \omega_{1} \omega_{2}\left(\omega_{1}+\omega_{2}\right)\left[a_{1} a_{2}\left(e^{-i\left(\omega_{1}+\omega_{2}\right) T}-1\right)-a_{1}^{*} a_{2}^{*}\left(e^{i\left(\omega_{1}+\omega_{2}\right) T}-1\right)\right] \\
& -i \gamma \omega_{1} \omega_{2}\left(\omega_{1}-\omega_{2}\right)\left[a_{1} a_{2}^{*}\left(e^{-i\left(\omega_{1}-\omega_{2}\right) T}-1\right)-a_{1}^{*} a_{2}\left(e^{i\left(\omega_{1}-\omega_{2}\right) T}-1\right)\right]
\end{aligned}
$$

we find that in the general path the Pais-Uhlenbeck action evaluates to

$$
I_{\mathrm{PU}}^{\mathrm{GEN}}=I_{\mathrm{STAT}}\left(a_{1}, a_{2}\right)+\frac{\gamma T}{4} \sum_{n=1}^{\infty} a_{n}^{2}\left[\frac{n^{4} \pi^{4}}{T^{4}}-\left(\omega_{1}^{2}+\omega_{2}^{2}\right) \frac{n^{2} \pi^{2}}{T^{2}}+3 \omega_{1}^{2} \omega_{2}^{2}\right] .
$$

From the form of Eq. (A8) we confirm that $q_{\mathrm{STAT}}(t)$ is indeed the stationary classical path $\left(\delta I_{\mathrm{PU}}^{\mathrm{GEN}} / \delta a_{n}\right.$ is zero when all $a_{n}$ are zero, even with the constraint of Eq. (Aquantum-mechanical path integral prescription for the initial to final overlap amplitude is thus given by

$$
\langle(q(T), \dot{q}(T)) \mid(q(0), \dot{q}(0))\rangle=\int_{-\infty}^{\infty} d a_{1} d a_{2} \ldots e^{(i / \hbar) I_{\mathrm{PU}}^{\mathrm{GEN}}}
$$


to then readily lead us (up to an overall $T$-dependent factor that we determine below) to a very familiar form for $\langle(q(T), \dot{q}(T)) \mid(q(0), \dot{q}(0))\rangle$, viz.

$$
\langle(q(T), \dot{q}(T)) \mid(q(0), \dot{q}(0))\rangle=e^{(i / \hbar) I_{\mathrm{STAT}}\left(a_{1}, a_{2}\right)} .
$$

For the phase space variation, if in $I_{\mathrm{HAM}}$ we replace $q$ by $q+\delta q, x$ by $x+\delta x, p_{q}$ by $p_{q}+\delta p_{q}$ and $p_{x}$ by $p_{x}+\delta p_{x}$, in first order in the variation we will obtain terms which depend on $\delta \dot{q}$ and $\delta \dot{x}$. In bringing these particular terms to a pure $\delta q$ and $\delta x$ form we will generate surface terms which depend on $\delta q$ and $\delta x$. Hence the classical Hamilton variational procedure requires that both $q$ and $x$ be held fixed at the end points, to thus nicely parallel the holding fixed of $q$ and $\dot{q}$ in the Euler-Lagrange variation described above. In terms of the stationary

$$
\begin{aligned}
& q_{\mathrm{STAT}}(t)=a_{1} e^{-i \omega_{1} t}+a_{2} e^{-i \omega_{2} t}+a_{1}^{*} e^{i \omega_{1} t}+a_{2}^{*} e^{i \omega_{2} t}, \\
& x_{\mathrm{STAT}}(t)=-i \omega_{1} a_{1} e^{-i \omega_{1} t}-i \omega_{2} a_{2} e^{-i \omega_{2} t}+i \omega_{1} a_{1}^{*} e^{i \omega_{1} t}+i \omega_{2} a_{2}^{*} e^{i \omega_{2} t}, \\
& p_{q}^{\mathrm{STAT}}(t)=i \gamma \omega_{1} \omega_{2}^{2} a_{1} e^{-i \omega_{1} t}+i \gamma \omega_{1}^{2} \omega_{2} a_{2} e^{-i \omega_{2} t}-i \gamma \omega_{1} \omega_{2}^{2} a_{1}^{*} e^{i \omega_{1} t}-i \gamma \omega_{1}^{2} \omega_{2} a_{2}^{*} e^{i \omega_{2} t}, \\
& p_{x}^{\mathrm{STAT}}(t)=-\gamma \omega_{1}^{2} a_{1} e^{-i \omega_{1} t}-\gamma \omega_{2}^{2} a_{2} e^{-i \omega_{2} t}-\gamma \omega_{1}^{2} a_{1}^{*} e^{i \omega_{1} t}-\gamma \omega_{2}^{2} a_{2}^{*} e^{i \omega_{2} t},
\end{aligned}
$$

we thus define the general phase space path to be of the form

$$
\begin{aligned}
& q_{\mathrm{GEN}}(t)=q_{\mathrm{STAT}}(t)+\sum_{n=1}^{\infty} a_{n} \sin \left(\frac{n \pi t}{T}\right), \\
& x_{\mathrm{GEN}}(t)=x_{\mathrm{STAT}}(t)+\sum_{n=1}^{\infty} b_{n} \sin \left(\frac{n \pi t}{T}\right), \\
& p_{q}^{\mathrm{GEN}}(t)=p_{q}^{\mathrm{STAT}}(t)+\sum_{n=1}^{\infty} c_{n} \cos \left(\frac{n \pi t}{T}\right)+c_{0}, \\
& p_{x}^{\mathrm{GEN}}(t)=p_{x}^{\mathrm{STAT}}(t)+\sum_{n=1}^{\infty} d_{n} \cos \left(\frac{n \pi t}{T}\right)+d_{0},
\end{aligned}
$$

with $q_{\mathrm{GEN}}(t)$ and $x_{\mathrm{GEN}}(t)$ respectively being equal to $q_{\mathrm{STAT}}(t)$ and $x_{\mathrm{STAT}}(t)$ at the end points, and with $p_{q}^{\mathrm{GEN}}(t)$ and $p_{x}^{\mathrm{GEN}}(t)$ not being constrained at the end points at all. On noting that we can rewrite $I_{\mathrm{HAM}}$ of Eq. (A2) in the form

$$
I_{\mathrm{HAM}}=\int_{0}^{T} d t\left[-\frac{1}{2 \gamma}\left(p_{x}-\gamma \dot{x}\right)^{2}+p_{q}(\dot{q}-x)+\frac{\gamma \dot{x}^{2}}{2}-\frac{\gamma}{2}\left(\omega_{1}^{2}+\omega_{2}^{2}\right) x^{2}+\frac{\gamma}{2} \omega_{1}^{2} \omega_{2}^{2} q^{2}\right]
$$

we find that in the general path $I_{\mathrm{HAM}}^{\mathrm{GEN}}$ evaluates to

$$
I_{\mathrm{HAM}}^{\mathrm{GEN}}=I_{\mathrm{STAT}}\left(a_{1}, a_{2}\right)-\frac{T d_{0}^{2}}{2 \gamma}-\frac{T}{4 \gamma} \sum_{n=1}^{\infty}\left(d_{n}-\frac{n \pi \gamma}{T} b_{n}\right)^{2}-\frac{2 T}{\pi} \sum_{n=\mathrm{odd}}^{\infty} \frac{c_{0} b_{n}}{n}+\frac{\pi}{2} \sum_{n=1}^{\infty} n c_{n} a_{n}
$$




$$
+\sum_{n, m \neq n} c_{n} b_{m} A_{n m}+\sum_{n=1}^{\infty}\left[\frac{\gamma n^{2} \pi^{2}}{4 T}-\frac{T \gamma\left(\omega_{1}^{2}+\omega_{2}^{2}\right)}{4}\right] b_{n}^{2}+\frac{T \gamma}{4} \omega_{1}^{2} \omega_{2}^{2} \sum_{n=1}^{\infty} a_{n}^{2}
$$

where $A_{n m}=\left[(-1)^{m-n}-1\right] / 2(m-n)+\left[(-1)^{m+n}-1\right] / 2(m+n)$ and $I_{\mathrm{STAT}}\left(a_{1}, a_{2}\right)$ is as given earlier (the Hamilton variation and the Euler-Lagrange variation yield the same stationary classical solution). Functional variation of $I_{\mathrm{HAM}}^{\mathrm{GEN}}$ with respect to $a_{n}, b_{n}, c_{n}$ and $d_{n}$ then confirms that $q_{\mathrm{STAT}}(t), x_{\mathrm{STAT}}(t), p_{q}^{\mathrm{STAT}}(t), p_{x}^{\mathrm{STAT}}(t)$ as given in Eq. (A11) is indeed the stationary solution to the Hamilton variation, with the phase space path integration then leading us (again up to an overall factor) right back to

$$
\langle(q(T), \dot{q}(T)) \mid(q(0), \dot{q}(0))\rangle=\int_{-\infty}^{\infty} d c_{0} d d_{0} \prod_{n=1}^{\infty} d a_{n} d b_{n} d c_{n} d d_{n} \ldots e^{(i / \hbar) I_{\mathrm{HAM}}^{\mathrm{GEN}}}=e^{(i / \hbar) I_{\mathrm{STAT}}\left(a_{1}, a_{2}\right)}
$$

just as it should.

Analogously, for the equal frequency theory the stationary classical solution is given by

$$
\begin{aligned}
& q_{\mathrm{STAT}}(t)=c_{1} e^{-i \omega t}+t c_{2} e^{-i \omega t}+c_{1}^{*} e^{i \omega t}+t c_{2}^{*} e^{i \omega t} \\
& x_{\mathrm{STAT}}(t)=-i \omega c_{1} e^{-i \omega t}+(1-i \omega t) c_{2} e^{-i \omega t}+i \omega c_{1}^{*} e^{i \omega t}+(1+i \omega t) c_{2}^{*} e^{i \omega t} \\
& p_{q}^{\mathrm{STAT}}(t)=i \gamma \omega^{3} c_{1} e^{-i \omega t}+\gamma \omega^{2}(1+i \omega t) c_{2} e^{-i \omega t}-i \gamma \omega^{3} c_{1}^{*} e^{i \omega t}+\gamma \omega^{2}(1-i \omega t) c_{2}^{*} e^{i \omega t} \\
& p_{x}^{\mathrm{STAT}}(t)=-\gamma \omega^{2} c_{1} e^{-i \omega t}-\gamma \omega(2 i+\omega t) c_{2} e^{-i \omega t}-\gamma \omega^{2} c_{1}^{*} e^{i \omega t}-\gamma \omega(-2 i+\omega t) c_{2}^{*} e^{i \omega t}
\end{aligned}
$$

with the stationary classical action evaluating to the form

$$
\begin{aligned}
I_{\mathrm{STAT}}\left(c_{1}, c_{2}\right)=\gamma \omega^{2} \int_{0}^{T} d t & {\left[2\left(\omega c_{1}+\omega t c_{2}+i c_{2}\right)^{2} e^{-2 i \omega t}+2\left(\omega c_{1}^{*}+\omega t c_{2}^{*}-i c_{2}^{*}\right)^{2} e^{2 i \omega t}\right.} \\
& \left.-\left(c_{2} e^{-i \omega t}-c_{2}^{*} e^{i \omega t}\right)^{2}\right],
\end{aligned}
$$

and with the quantum-mechanical overlap evaluating to

$$
\langle(q(T), \dot{q}(T)) \mid(q(0), \dot{q}(0))\rangle=e^{(i / \hbar) I_{\mathrm{STAT}}\left(c_{1}, c_{2}\right)}
$$

just as it should.

Having now solved for both the unequal and equal frequency Pais-Uhlenbeck theories, it is instructive to compare the structure that we have found for each of the two cases with that associated with a standard second order harmonic oscillator theory. For a single harmonic oscillator with classical action and stationary classical solution

$$
S_{\mathrm{SINGLE}}=\frac{m}{2} \int_{0}^{T} d t\left[\dot{q}^{2}-\omega^{2} q^{2}\right] \quad, \quad q_{\mathrm{STAT}}(t)=d e^{-i \omega t}+d^{*} e^{i \omega t}
$$


we recall that the stationary classical action and quantum-mechanical overlap amplitude evaluate to

$$
I_{\mathrm{STAT}}(d)=-m \omega^{2} \int_{0}^{T} d t\left[d^{2} e^{-2 i \omega t}+d^{* 2} e^{2 i \omega t}\right],\langle q(T) \mid q(0)\rangle=e^{(i / \hbar) I_{\mathrm{STAT}}(d)}
$$

with there being no $d d^{*}$ cross terms. To compare with the unequal frequency Pais-Uhlenbeck theory, on noting the kinematic identity

$$
\begin{aligned}
& {\left[\left(\frac{d}{d t}-\omega_{1}\right)\left(\frac{d^{2}}{d t^{2}}+\omega_{2}^{2}\right) q\right]\left[\left(\frac{d}{d t}+\omega_{1}\right)\left(\frac{d^{2}}{d t^{2}}+\omega_{2}^{2}\right) q\right]} \\
& -\left[\left(\frac{d}{d t}-\omega_{2}\right)\left(\frac{d^{2}}{d t^{2}}+\omega_{1}^{2}\right) q\right]\left[\left(\frac{d}{d t}+\omega_{2}\right)\left(\frac{d^{2}}{d t^{2}}+\omega_{1}^{2}\right) q\right] \\
& =\left(\omega_{1}^{2}-\omega_{2}^{2}\right)\left[\ddot{q}^{2}-\left(\omega_{1}^{2}+\omega_{2}^{2}\right) \dot{q}^{2}+\omega_{1}^{2} \omega_{2}^{2} q^{2}-2 \frac{d}{d t}(\dot{q} \ddot{q})\right]
\end{aligned}
$$

and recognizing that $d^{2} / d t^{2}+\omega_{1}^{2}$ and $d^{2} / d t^{2}+\omega_{2}^{2}$ act as the projector operators

$$
\begin{aligned}
& \left(\frac{d^{2}}{d t^{2}}+\omega_{1}^{2}\right) q_{\mathrm{STAT}}(t)=\left(\omega_{1}^{2}-\omega_{2}^{2}\right)\left(a_{2} e^{-i \omega_{2} t}+a_{2}^{*} e^{i \omega_{2} t}\right), \\
& \left(\frac{d^{2}}{d t^{2}}+\omega_{2}^{2}\right) q_{\mathrm{STAT}}(t)=\left(\omega_{2}^{2}-\omega_{1}^{2}\right)\left(a_{1} e^{-i \omega_{1} t}+a_{1}^{*} e^{i \omega_{1} t}\right),
\end{aligned}
$$

we see that the unequal frequency stationary classical action of Eq. (A7) can be reexpressed as

$$
\begin{aligned}
I_{\mathrm{STAT}}\left(a_{1}, a_{2}\right) & =-\gamma\left(\omega_{1}^{2}-\omega_{2}^{2}\right) \int_{0}^{T} d t\left[\omega_{1}^{2}\left(a_{1}^{2} e^{-2 i \omega_{1} t}+a_{1}^{* 2} e^{2 i \omega_{1} t}\right)-\omega_{2}^{2}\left(a_{2}^{2} e^{-2 i \omega_{2} t}+a_{2}^{* 2} e^{2 i \omega_{2} t}\right)\right] \\
& +\gamma\left[\dot{q}_{\mathrm{STAT}}(T) \ddot{q}_{\mathrm{STAT}}(T)-\dot{q}_{\mathrm{STAT}}(0) \ddot{q}_{\mathrm{STAT}}(0)\right] .
\end{aligned}
$$

Similarly, in the equal frequency case [obtainable directly from Eq. (A23) by setting $a_{1}=$ $(1 / 2)\left(c_{1}+i c_{2} / \epsilon\right), a_{2}=(1 / 2)\left(c_{1}-i c_{2} / \epsilon\right), \omega_{1}=\omega+\epsilon, \omega_{2}=\omega-\epsilon$, and then taking the $\epsilon \rightarrow 0$ limit, a limit in which $q(\epsilon \neq 0)$ of Eq. (48) continues smoothly into $q(\epsilon=0)$ of Eq. (54) since $\left.a_{1} e^{-i \omega_{1} t}+a_{2} e^{-i \omega_{2} t} \rightarrow e^{-i \omega t}\left(c_{1}+c_{2} t\right)+O\left(\epsilon^{2}\right)\right]$, the stationary classical action is given by

$$
\begin{aligned}
I_{\mathrm{STAT}}\left(c_{1}, c_{2}\right) & =4 \omega^{2} \gamma \int_{0}^{T} d t\left[\left(c_{2}^{2}-i \omega t c_{2}^{2}-i \omega c_{1} c_{2}\right) e^{-2 i \omega t}+\left(c_{2}^{* 2}+i \omega t c_{2}^{* 2}+i \omega c_{1}^{*} c_{2}^{*}\right) e^{2 i \omega t}\right] \\
& +\gamma\left[\dot{q}_{\mathrm{STAT}}(T) \ddot{q}_{\mathrm{STAT}}(T)-\dot{q}_{\mathrm{STAT}}(0) \ddot{q}_{\mathrm{STAT}}(0)\right]
\end{aligned}
$$

Apart from surface terms then, we see that the unequal frequency action $I_{\mathrm{STAT}}\left(a_{1}, a_{2}\right)$ of Eq. (A23) has the form of a difference between two Eq. (A20) type oscillators, while the equal frequency $I_{\mathrm{STAT}}\left(c_{1}, c_{2}\right)$ of Eq. (A24) possesses no $c_{1}^{2}$ or $c_{1}^{* 2}$ type terms and has a form similar 
to that of just a single harmonic oscillator. Our study of path integral quantization of the Pais-Uhlenbeck theory thus reflects the structure of both the classical [recall the structure of $H_{\mathrm{STAT}}\left(\omega_{1} \neq \omega_{2}\right)$ of Eq. (31) and $H_{\mathrm{STAT}}\left(\omega_{1}=\omega_{2}=\omega\right)$ of Eq. (33)] and the second-quantized Hamiltonian based analyses we presented earlier where the unequal frequency theory was found to possess two sets of energy eigenstates (one normal and the other ghost), while the equal frequency theory was found to possess just a normal one.

To explore and elucidate further the similarity between the structures of the first- and second-quantized formulations of the fourth order theory, it is instructive to actually perform the integration required for Eq. (A23), with use of Eq. (A3) enabling us to substitute for $\ddot{q}_{\mathrm{STAT}}(T), a_{2}$ and $a_{2}^{*}$ and rewrite Eq. (A23) in the form

$$
\begin{aligned}
I_{\mathrm{STAT}}\left(a_{1}, a_{2}\right)= & -\frac{\gamma}{2}\left(\omega_{1}^{2}+\omega_{2}^{2}\right) q_{\mathrm{STAT}}(T) \dot{q}_{\mathrm{STAT}}(T)-\frac{\gamma}{2}\left(\omega_{1}^{2}-\omega_{2}^{2}\right) \dot{q}_{\mathrm{STAT}}(T)\left(a_{1} e^{-i \omega_{1} T}+a_{1}^{*} e^{i \omega_{1} T}\right) \\
& -\frac{i \gamma}{2} \omega_{1}\left(\omega_{1}^{2}-\omega_{2}^{2}\right) q_{\mathrm{STAT}}(T)\left(a_{1} e^{-i \omega_{1} T}-a_{1}^{*} e^{i \omega_{1} T}\right) \quad-(T=0) . \quad \text { (A25) }
\end{aligned}
$$

To eliminate the dependence on $a_{1}$ and $a_{1}^{*}$, we need to invert Eq. (A3) and its time derivative, to find in terms of $q_{i}=q_{\mathrm{STAT}}(0), \dot{q}_{i}=\dot{q}_{\mathrm{STAT}}(0), q_{f}=q_{\mathrm{STAT}}(T), \dot{q}_{f}=\dot{q}_{\mathrm{STAT}}(T)$, that $a_{1}$ is given as

$$
\begin{aligned}
Y(T) a_{1}= & \left.\omega_{2} q_{i}\left[2 \omega_{1}-\left(\omega_{1}-\omega_{2}\right) e^{i\left(\omega_{1}+\omega_{2}\right) T}-\left(\omega_{1}+\omega_{2}\right) e^{i\left(\omega_{1}-\omega_{2}\right) T}\right)\right] \\
& \left.+i \dot{q}_{i}\left[2 \omega_{2}+\left(\omega_{1}-\omega_{2}\right) e^{i\left(\omega_{1}+\omega_{2}\right) T}-\left(\omega_{1}+\omega_{2}\right) e^{i\left(\omega_{1}-\omega_{2}\right) T}\right)\right] \\
& +\omega_{2} q_{f}\left[2 \omega_{1} e^{i \omega_{1} T}-\left(\omega_{1}-\omega_{2}\right) e^{-i \omega_{2} T}-\left(\omega_{1}+\omega_{2}\right) e^{i \omega_{2} T}\right] \\
& +i \dot{q}_{f}\left[2 \omega_{2} e^{i \omega_{1} T}+\left(\omega_{1}-\omega_{2}\right) e^{-i \omega_{2} T}-\left(\omega_{1}+\omega_{2}\right) e^{i \omega_{2} T}\right]
\end{aligned}
$$

(and analogously for $a_{1}^{*}$ ), where the function $Y(T)$ that we have introduced here is given by

$$
\begin{aligned}
Y(T)=8 \omega_{1} \omega_{2} & +\left(\omega_{1}-\omega_{2}\right)^{2}\left(e^{-i\left(\omega_{1}+\omega_{2}\right) T}+e^{i\left(\omega_{1}+\omega_{2}\right) T}\right) \\
& -\left(\omega_{1}+\omega_{2}\right)^{2}\left(e^{-i\left(\omega_{1}-\omega_{2}\right) T}+e^{i\left(\omega_{1}-\omega_{2}\right) T}\right) .
\end{aligned}
$$

Given Eq. (A26), the unequal frequency Eq. (A25) is then found to take the form $I_{\mathrm{STAT}}\left(a_{1}, a_{2}\right)=-\frac{\gamma}{2}\left(\omega_{1}^{2}+\omega_{2}^{2}\right) q_{f} \dot{q}_{f}+\frac{\gamma}{2}\left(\omega_{1}^{2}+\omega_{2}^{2}\right) q_{i} \dot{q}_{i}-\frac{\gamma}{2}\left(\omega_{1}^{2}-\omega_{2}^{2}\right) \frac{\left[X_{f}(T)+X_{i}(T)+X_{i f}(T)\right]}{Y(T)}$,

where $X_{f}(T)$ is given by

$$
\begin{aligned}
X_{f}(T)= & -i \omega_{1} \omega_{2} q_{f}^{2}\left[\left(\omega_{1}-\omega_{2}\right)\left(e^{-i\left(\omega_{1}+\omega_{2}\right) T}-e^{i\left(\omega_{1}+\omega_{2}\right) T}\right)+\left(\omega_{1}+\omega_{2}\right)\left(e^{-i\left(\omega_{1}-\omega_{2}\right) T}-e^{i\left(\omega_{1}-\omega_{2}\right) T}\right)\right] \\
& +i \dot{q}_{f}^{2}\left[\left(\omega_{1}-\omega_{2}\right)\left(e^{-i\left(\omega_{1}+\omega_{2}\right) T}-e^{i\left(\omega_{1}+\omega_{2}\right) T}\right)-\left(\omega_{1}+\omega_{2}\right)\left(e^{-i\left(\omega_{1}-\omega_{2}\right) T}-e^{i\left(\omega_{1}-\omega_{2}\right) T}\right)\right] \\
& -q_{f} \dot{q}_{f}\left(\omega_{1}^{2}-\omega_{2}^{2}\right)\left[e^{-i\left(\omega_{1}+\omega_{2}\right) T}+e^{i\left(\omega_{1}+\omega_{2}\right) T}-e^{-i\left(\omega_{1}-\omega_{2}\right) T}-e^{i\left(\omega_{1}-\omega_{2}\right) T}\right], \quad \text { (A29) }
\end{aligned}
$$


$X_{i}(t)$ is given by

$$
\begin{aligned}
X_{i}(T)= & -i \omega_{1} \omega_{2} q_{i}^{2}\left[\left(\omega_{1}-\omega_{2}\right)\left(e^{-i\left(\omega_{1}+\omega_{2}\right) T}-e^{i\left(\omega_{1}+\omega_{2}\right) T}\right)+\left(\omega_{1}+\omega_{2}\right)\left(e^{-i\left(\omega_{1}-\omega_{2}\right) T}-e^{i\left(\omega_{1}-\omega_{2}\right) T}\right)\right] \\
& +i \dot{q}_{i}^{2}\left[\left(\omega_{1}-\omega_{2}\right)\left(e^{-i\left(\omega_{1}+\omega_{2}\right) T}-e^{i\left(\omega_{1}+\omega_{2}\right) T}\right)-\left(\omega_{1}+\omega_{2}\right)\left(e^{-i\left(\omega_{1}-\omega_{2}\right) T}-e^{i\left(\omega_{1}-\omega_{2}\right) T}\right)\right] \\
& +q_{i} \dot{q}_{i}\left(\omega_{1}^{2}-\omega_{2}^{2}\right)\left[e^{-i\left(\omega_{1}+\omega_{2}\right) T}+e^{i\left(\omega_{1}+\omega_{2}\right) T}-e^{-i\left(\omega_{1}-\omega_{2}\right) T}-e^{i\left(\omega_{1}-\omega_{2}\right) T}\right], \quad \text { (A30) }
\end{aligned}
$$

and $X_{i f}(T)$ is given by

$$
\begin{aligned}
X_{i f}(T) & =4 i \omega_{2} e^{-i \omega_{1} T}\left(\dot{q}_{i}-i \omega_{1} q_{i}\right)\left(\dot{q}_{f}+i \omega_{1} q_{f}\right)-4 i \omega_{2} e^{i \omega_{1} T}\left(\dot{q}_{i}+i \omega_{1} q_{i}\right)\left(\dot{q}_{f}-i \omega_{1} q_{f}\right) \\
& -4 i \omega_{1} e^{-i \omega_{2} T}\left(\dot{q}_{i}-i \omega_{2} q_{i}\right)\left(\dot{q}_{f}+i \omega_{2} q_{f}\right)+4 i \omega_{1} e^{i \omega_{2} T}\left(\dot{q}_{i}+i \omega_{2} q_{i}\right)\left(\dot{q}_{f}-i \omega_{2} q_{f}\right)
\end{aligned}
$$

Similarly, for the equal frequency case, the stationary classical action $I_{\mathrm{STAT}}\left(a_{1}, a_{2}, \epsilon=0\right)$ (obtainable directly from Eq. (A28) by taking the $\epsilon \rightarrow 0$ limit) can be reexpressed as

$$
I_{\mathrm{STAT}}\left(a_{1}, a_{2}, \epsilon=0\right)=-\gamma \omega^{2} q_{f} \dot{q}_{f}+\gamma \omega^{2} q_{i} \dot{q}_{i}-\gamma \omega \frac{\left[\hat{X}_{f}(T)+\hat{X}_{i}(T)+\hat{X}_{i f}(T)\right]}{\hat{Y}(T)}
$$

where $\hat{X}_{f}(T)$ is given by

$$
\begin{aligned}
\hat{X}_{f}(T)= & -i \omega^{2} q_{f}^{2}\left(e^{-2 i \omega T}-e^{2 i \omega T}-4 i \omega T\right)+i \dot{q}_{f}^{2}\left(e^{-2 i \omega T}-e^{2 i \omega T}+4 i \omega T\right) \\
& -2 \omega q_{f} \dot{q}_{f}\left(e^{-2 i \omega T}+e^{2 i \omega T}-2\right),
\end{aligned}
$$

$\hat{X}_{i}(t)$ is given by

$$
\begin{aligned}
\hat{X}_{i}(T)= & -i \omega^{2} q_{i}^{2}\left(e^{-2 i \omega T}-e^{2 i \omega T}-4 i \omega T\right)+i \dot{q}_{i}^{2}\left(e^{-2 i \omega T}-e^{2 i \omega T}+4 i \omega T\right) \\
& +2 \omega q_{i} \dot{q}_{i}\left(e^{-2 i \omega T}+e^{2 i \omega T}-2\right)
\end{aligned}
$$

$\hat{X}_{i f}(T)$ is given by

$$
\begin{aligned}
\hat{X}_{i f}(T) & =4 \omega T e^{-i \omega T}\left(\dot{q}_{i}-i \omega q_{i}\right)\left(\dot{q}_{f}+i \omega q_{f}\right)+4 \omega T e^{i \omega T}\left(\dot{q}_{i}+i \omega q_{i}\right)\left(\dot{q}_{f}-i \omega q_{f}\right) \\
& -4 i\left(e^{-i \omega T}-e^{i \omega T}\right)\left(\dot{q}_{i} \dot{q}_{f}-\omega^{2} q_{i} q_{f}\right)
\end{aligned}
$$

and $\hat{Y}(T)$ is given by

$$
\hat{Y}(T)=e^{-2 i \omega T}+e^{2 i \omega T}+4 \omega^{2} T^{2}-2 .
$$

Now that we have $I_{\text {STAT }}\left(a_{1}, a_{2}\right)$ in a form in which it is written entirely in terms of end point values, we can use the artifice of going to Euclidean time by setting $T=-i \tau$, with 
the $\tau \rightarrow \infty$ limit of Eqs. (A28) and Eq. (A32) then yielding a leading term for the unequal frequency theory overlap amplitude of the form

$$
\begin{aligned}
\langle(q(-i \tau), \dot{q}(-i \tau)) \mid(q(0), \dot{q}(0))\rangle & \rightarrow \exp \left[\frac{\gamma}{2 \hbar} \omega_{1} \omega_{2}\left(\omega_{1}+\omega_{2}\right) q_{i}^{2}-\frac{i \gamma}{\hbar} \omega_{1} \omega_{2} q_{i} \dot{q}_{i}-\frac{\gamma}{2 \hbar}\left(\omega_{1}+\omega_{2}\right) \dot{q}_{i}^{2}\right] \\
& \times \exp \left[\frac{\gamma}{2 \hbar} \omega_{1} \omega_{2}\left(\omega_{1}+\omega_{2}\right) q_{f}^{2}+\frac{i \gamma}{\hbar} \omega_{1} \omega_{2} q_{f} \dot{q}_{f}-\frac{\gamma}{2 \hbar}\left(\omega_{1}+\omega_{2}\right) \dot{q}_{f}^{2}\right]
\end{aligned}
$$

and a leading term for the equal frequency overlap amplitude of the form

$$
\begin{aligned}
\langle(q(-i \tau), \dot{q}(-i \tau)) \mid(q(0), \dot{q}(0))\rangle & \rightarrow \exp \left[\frac{\gamma}{\hbar} \omega^{3} q_{i}^{2}-\frac{i \gamma}{\hbar} \omega^{2} q_{i} \dot{q}_{i}-\frac{\gamma}{\hbar} \omega \dot{q}_{i}^{2}\right] \\
& \times \exp \left[\frac{\gamma}{\hbar} \omega^{3} q_{f}^{2}+\frac{i \gamma}{\hbar} \omega^{2} q_{f} \dot{q}_{f}-\frac{\gamma}{\hbar} \omega \dot{q}_{f}^{2}\right]
\end{aligned}
$$

For comparison purposes, we note that for the second order harmonic oscillator of Eqs. (A19) and (A20), the stationary classical action $I_{\mathrm{STAT}}(d)$ evaluates to

$$
I_{\mathrm{STAT}}(d)=\frac{m \omega}{2 \sin (\omega T)}\left[\cos (\omega T)\left(q_{i}^{2}+q_{f}^{2}\right)-2 q_{i} q_{f}\right]
$$

with the Euclidean overlap amplitude then asymptoting to

$$
\langle q(-i \tau) \mid q(0)\rangle \rightarrow \exp \left[-\frac{m \omega q_{i}^{2}}{2 \hbar}\right] \exp \left[-\frac{m \omega q_{f}^{2}}{2 \hbar}\right] .
$$

With the right-hand side of Eq. (A40) immediately revealing the familiar ground state wave function of the second order harmonic oscillator (just as required of the $\tau \rightarrow \infty$ limit where $e^{-i E T / \hbar}=e^{-E \tau / \hbar}$ is dominated by the lowest energy eigenvalue), we can thus anticipate that Eqs. (A37) and (A38) are therefore providing us with the ground state wave functions of the unequal and equal frequency fourth order Pais-Uhlenbeck oscillator theories, with the Pais-Uhlenbeck theory being seen to actually have a well-defined ground state in both the unequal and equal frequency cases and thus not be unbounded from below. And so to check this expectation, we analyze the Pais-Uhlenbeck theory from the perspective of first-quantized wave mechanics, an exercise which is anyway of interest in its own right.

\section{APPENDIX B: WAVE MECHANICS FOR THE FOURTH ORDER THEORY}

In addition to the second-quantized Fock space and path integral quantization treatments

of the fourth order theory, it is also of interest to analyze the theory from the perspective of 
first-quantized wave mechanics. Specifically, instead of realizing the $\left[q, p_{q}\right]=i \hbar,\left[x, p_{x}\right]=i \hbar$ commutators via creation and annihilation operators, we instead realize them as derivative operators

$$
p_{q}=-i \hbar \frac{\partial}{\partial q}, p_{x}=-i \hbar \frac{\partial}{\partial x}
$$

which act in an effective two-dimensional coordinate space with coordinates $q$ and $x$ [22]. In such a space the Schrödinger equation associated with the unequal frequency theory Hamiltonian of Eq. (22) takes the form

$$
H_{\mathrm{SCHR}} \psi_{n}(q, x)=\left[-\frac{\hbar^{2}}{2 \gamma} \frac{\partial^{2}}{\partial x^{2}}-i \hbar x \frac{\partial}{\partial q}+\frac{\gamma}{2}\left(\omega_{1}^{2}+\omega_{2}^{2}\right) x^{2}-\frac{\gamma}{2} \omega_{1}^{2} \omega_{2}^{2} q^{2}\right] \psi_{n}(q, x)=E_{n} \psi_{n}(q, x) .
$$

To find the $\psi_{0}(x, y)$ analog of the second-quantized state $|\Omega\rangle$ which the $a_{1}$ and $a_{2}$ operators annihilate, we recall from Eq. (39) that the relation between the $a_{1}, a_{2}, a_{1}^{\dagger}$ and $a_{2}^{\dagger}$ operators and the $q, x, p_{q}$ and $p_{x}$ operators is a linear one, with the coordinate space analogs of $a_{1}|\Omega\rangle=$ $0, a_{2}|\Omega\rangle=0$ then being linear relations of the form $\left(e_{1} \partial / \partial q+e_{2} \partial / \partial x+e_{3} q+e_{4} x\right) \psi_{0}(x, y)=0$ with appropriate coefficients $e_{1}, e_{2}, e_{3}$ and $e_{4}$. Consequently, $\psi_{0}(x, y)$ must be in the form of an exponential whose exponent is a linear combination of $q^{2}, q x$ and $x^{2}$ terms with appropriate coefficients. With the energy of the state $|\Omega\rangle$ being the zero point energy $(\hbar / 2)\left(\omega_{1}+\omega_{2}\right)$ as given Eq. (41), we thus solve Eq. (B2) with $E_{0}=(\hbar / 2)\left(\omega_{1}+\omega_{2}\right)$, to find directly that $\psi_{0}(q, x)$ is given by

$$
\psi_{0}(q, x)=\exp \left[\frac{\gamma}{2 \hbar} \omega_{1} \omega_{2}\left(\omega_{1}+\omega_{2}\right) q^{2}+\frac{i \gamma}{\hbar} \omega_{1} \omega_{2} q x-\frac{\gamma}{2 \hbar}\left(\omega_{1}+\omega_{2}\right) x^{2}\right] .
$$

Since the one-particle Fock space states which lie at $\hbar \omega_{1}$ and $\hbar \omega_{2}$ above the ground state are obtained from $|\Omega\rangle$ by the actions of $a_{1}^{\dagger}$ and $a_{2}^{\dagger}$ on it, in coordinate space this has the effect of multiplying $\psi_{0}(q, x)$ by a linear combination of $q$ and $x$. The needed coefficients are readily fixed from Eq. (B2), and yield excited states of the form

$$
\begin{aligned}
& \psi_{1}(q, x)=\left(x-i \omega_{2} q\right) \psi_{0}(q, x), \quad E_{1}=E_{0}+\hbar \omega_{1} \\
& \psi_{2}(q, x)=\left(x-i \omega_{1} q\right) \psi_{0}(q, x), \quad E_{2}=E_{0}+\hbar \omega_{2} .
\end{aligned}
$$

Similarly, since the two-particle Fock space states which lie at $2 \hbar \omega_{1}, \hbar\left(\omega_{1}+\omega_{2}\right)$ and $2 \hbar \omega_{2}$ above the ground state are obtained from $|\Omega\rangle$ by the actions of $a_{1}^{\dagger 2}, a_{1}^{\dagger} a_{2}^{\dagger}$ and $a_{2}^{\dagger 2}$ on it, in coordinate space this has the effect of multiplying $\psi_{0}(q, x)$ by a linear combination of $q^{2}, q x$, 
$x^{2}$ and a constant, with the relevant excited states being found to be of the form

$$
\begin{aligned}
& \psi_{3}(q, x)=\left[\left(x-i \omega_{2} q\right)^{2}-\frac{1}{2 \gamma \omega_{1}}\right] \psi_{0}(q, x), E_{3}=E_{0}+2 \hbar \omega_{1} \\
& \psi_{4}(q, x)=\left[\left(x-i \omega_{1} q\right)\left(x-i \omega_{2} q\right)-\frac{1}{\gamma\left(\omega_{1}+\omega_{2}\right)}\right] \psi_{0}(q, x), E_{4}=E_{0}+\hbar\left(\omega_{1}+\omega_{2}\right) \\
& \psi_{5}(q, x)=\left[\left(x-i \omega_{1} q\right)^{2}-\frac{1}{2 \gamma \omega_{2}}\right] \psi_{0}(q, x), \quad E_{5}=E_{0}+2 \hbar \omega_{2}
\end{aligned}
$$

While we have thus found the first-quantized family of states we seek (and even recovered our earlier result that in the equal frequency limit states such as $\psi_{1}(q, x)$ and $\psi_{1}(q, x)$ collapse into one and the same state, a limit in which $H_{\mathrm{SCHR}}$ of Eq. (B2) is continuous), we note that the wave equation of Eq. (B2) is symmetric under $\omega_{1} \rightarrow-\omega_{1}, \omega_{2} \rightarrow-\omega_{2}$, with the state

$$
\hat{\psi}_{0}(q, x)=\exp \left[-\frac{\gamma}{2 \hbar} \omega_{1} \omega_{2}\left(\omega_{1}+\omega_{2}\right) q^{2}+\frac{i \gamma}{\hbar} \omega_{1} \omega_{2} q x+\frac{\gamma}{2 \hbar}\left(\omega_{1}+\omega_{2}\right) x^{2}\right]
$$

immediately being recognized as being an energy state with negative energy $\hat{E}_{0}=-E_{0}=$ $-(\hbar / 2)\left(\omega_{1}+\omega_{2}\right)$. Moreover, this same pattern persists for the multi-particle states as well causing the Schrödinger Hamiltonian $H_{\mathrm{SCHR}}$ of Eq. (B2) to appear to be unbounded from below. As such, this family of first-quantized states with negative energies corresponds to building second-quantized states out of a Fock state $|\hat{\Omega}\rangle$ that $a_{1}^{\dagger}$ and $a_{2}^{\dagger}$ annihilate, with it being $a_{1}$ and $a_{2}$ which are to then serve as the creation operators, with the Hamiltonian of Eq. (41) being rewritable in the form

$$
H=2 \hbar \gamma\left(\omega_{1}^{2}-\omega_{2}^{2}\right)\left(\omega_{1}^{2} a_{1} a_{1}^{\dagger}-\omega_{2}^{2} a_{2} a_{2}^{\dagger}\right)-\frac{\hbar}{2}\left(\omega_{1}+\omega_{2}\right)
$$

with a now negative zero point energy.

To assess the significance of this family of negative energy states associated with $H_{\mathrm{SCHR}}$, we recall the situation which holds for the standard one-dimensional simple harmonic oscillator where the Schrödinger equation is of the form

$$
\left[-\frac{\hbar^{2}}{2 m} \frac{d^{2}}{d q^{2}}+\frac{m \omega^{2}}{2}\right] \psi(q)=E \psi(q)
$$

As well as the familiar spatially convergent state $\psi_{0}(q)=e^{-m \omega q^{2} / 2 \hbar}$ with energy $E_{0}=\hbar \omega / 2$, as a differential equation Eq. (Bי (B) also possesses an exact solution of the spatially divergent form $\hat{\psi}_{0}(q)=e^{m \omega q^{2} / 2 \hbar}$ with an energy $\hat{E}_{0}=-\hbar \omega / 2$ which is negative, and indeed this latter solution is in the sector of the theory associated with treating $a^{\dagger}$ as the annihilator (of a state 
$|\hat{\Omega}\rangle)$ and $a$ as the creator in the Hamiltonian $H=\hbar \omega\left(a^{\dagger} a+1 / 2\right)=\hbar \omega\left(a a^{\dagger}-1 / 2\right)$. While both families of solutions (the positive and the negative energy sets, the $a^{\dagger}|\Omega\rangle$ with positive norm and the $a|\hat{\Omega}\rangle$ with negative norm) occur in the first-quantized theory, the two families cannot simultaneously occur in a second-quantized formulation of the theory since we can build a Fock space vacuum out of states which either $a$ or $a^{\dagger}$ annihilate but not out of one which they both do. To determine which of these two sectors is the relevant one for the one-dimensional oscillator theory, we recall the path integral formulation given in appendix A where the infinite Euclidean time limit of the overlap amplitude led us unambiguously to the wave function which appears in Eq. (A40), viz. to the one which is associated with the positive frequency zero point energy. The $T=-i \tau$ limit of the path integral thus automatically leads us to the sector of the theory which is bounded from below. Consequently, if we now apply the same Euclidean time procedure to the fourth order theory, we see that the limit of Eq. (A37) leads us automatically to none other than the positive energy wave function $\psi_{0}(q, x)$ given in Eq. (B3) and not at all to the negative energy wave function $\hat{\psi}_{0}(q, x)$ which is given in Eq. ( $(\underline{\mathrm{B} 6})$. The path integral determination of the overlap amplitude $\langle(q(T), \dot{q}(T)) \mid(q(0), \dot{q}(0))\rangle$ as given in Eq. (A10) thus reveals that just like the second order oscillator, the unequal frequency fourth order Pais-Uhlenbeck oscillator is also bounded from below, with the negative energy solutions to Eq. (B2) not being of relevance to the theory. The unequal frequency Pais-Uhlenbeck theory is thus quite well-behaved. And with there being no negative energy eigenstate contributions to the path integral, the result of [3] can thus to be understood as the statement that interactions which are themselves bounded will not lead to transitions between the positive and negative energy eigenstates. Finally, with the wave functions which appear on the right-hand side of Eq. (A37) continuing directly into those on the right hand side of Eq. (A38) in the equal frequency limit, we can conclude that the equal frequency theory is bounded from below as well, just as we found earlier when we constructed its second-quantized energy spectrum.

As well as obtain the ground state wave function $\psi_{0}(q, x)$ of the Pais-Uhlenbeck theory via path integration, to be fully assured that path integral quantization is indeed giving $\psi_{0}(q, x)$ as the true ground state of the theory, we need to recover the zero point energy from the path integral as well. To this end we need to determine the overall $T$-independent factor which is to multiply the right-hand side of Eq. (A10). To fix this factor we take advantage of the fact that the overlap integral $\langle(q(T), \dot{q}(T)) \mid(q(0), \dot{q}(0))\rangle$ can also be interpreted as a wave 
function $\psi(q, x, t)$ which obeys the Schrödinger equation $i \hbar \partial_{t} \psi(q, x, t)=H_{\mathrm{SCHR}} \psi(q, x, t)$ associated with the Hamiltonian which is given in Eq. (B22) 23]. On thus imposing the Schrödinger equation we find that $\langle(q(T), \dot{q}(T)) \mid(q(0), \dot{q}(0))\rangle$ will indeed obey it if Eq. (A10) is normalized as

$$
\langle(q(T), \dot{q}(T)) \mid(q(0), \dot{q}(0))\rangle=Y^{-1 / 2}(T) e^{(i / \hbar) I_{\mathrm{STAT}}\left(a_{1}, a_{2}\right)}
$$

where $Y(T)$ is the function given in Eq. (A27) 24]. Then, with the asymptotic Euclidean limit of $Y^{-1 / 2}(T)$ being none other than $e^{-\left(\omega_{1}+\omega_{2}\right) \tau / 2}$, the requisite zero point energy is recovered just as desired. Similarly, in the equal frequency case the relevant normalization factor is found to be given by $\hat{Y}^{-1 / 2}(T)$ where $\hat{Y}(T)$ is given in Eq. (A36), with the asymptotic Euclidean limit of $\hat{Y}^{-1 / 2}(T)$ being precisely $e^{-\omega \tau}$, again just as desired.

In addition to obtaining the ground state wave function, it is also instructive to use the the path integral formulation to extract the first excited states as well. To do thus we need to identify the first non-leading terms in the $\tau \rightarrow \infty$ limit of $\langle(q(T), \dot{q}(T)) \mid(q(0), \dot{q}(0))\rangle$, and directly find from Eq. (A28) that Eq. (A37) is now extended to

$$
\begin{aligned}
& \langle(q(-i \tau), \dot{q}(-i \tau)) \mid(q(0), \dot{q}(0))\rangle \rightarrow \psi_{0}^{*}\left(q_{i}, x_{i}\right) \psi_{0}\left(q_{f}, x_{f}\right) e^{-\left(\omega_{1}+\omega_{2}\right) \tau / 2} \\
& +\frac{2 \gamma e^{-\left(\omega_{1}+\omega_{2}\right) \tau / 2}}{\hbar}\left(\frac{\omega_{1}+\omega_{2}}{\omega_{1}-\omega_{2}}\right)\left[\omega_{1} \psi_{1}^{*}\left(q_{i}, x_{i}\right) \psi_{1}\left(q_{f}, x_{f}\right) e^{-\omega_{1} \tau}-\omega_{2} \psi_{2}^{*}\left(q_{i}, x_{i}\right) \psi_{2}\left(q_{f}, x_{f}\right) e^{-\omega_{2} \tau}\right]
\end{aligned}
$$

where the wave functions are precisely as given in Eqs. (B3) and (B4). As well as recover the fact that the wave functions of Eq. (Bי states, of even more interest is to note that in Eq. (B10) there is a relative minus sign between the wave functions. The path integral is thus signaling that one of the two states would have to have ghost signature, just as we had found earlier in our second quantization study of the unequal frequency Pais-Uhlenbeck theory. Despite the presence of the ghost signature, as we had noted earlier, in the presence of bounded interactions, the path integral would remain well-behaved, with the positive and negative signatured states not being able to combine into states of negative energy and cascade into oblivion. Finally, as regards the equal frequency case, we note that when Eq. (A32) is expanded in powers of $e^{-\omega \tau}$, the two opposite signatured terms combine into one term with $\psi_{1}(q, x)=\psi_{2}(q, x)$ [just as needed to cancel the $1 /\left(\omega_{1}-\omega_{2}\right)$ singularity in Eq. (B10)], with Eq. (A38) being extended to

$$
\langle(q(-i \tau), \dot{q}(-i \tau)) \mid(q(0), \dot{q}(0))\rangle \rightarrow \psi_{0}^{*}\left(q_{i}, x_{i}\right) \psi_{0}\left(q_{f}, x_{f}\right) e^{-\omega \tau}
$$




$$
-\frac{4 \gamma \omega^{2} \tau e^{-\omega \tau}}{\hbar} \psi_{1}^{*}\left(q_{i}, x_{i}\right) \psi_{1}\left(q_{f}, x_{f}\right) e^{-\omega \tau}
$$

and with the equal frequency theory thus possessing just a single one-particle state and not two, precisely as we had found previously.

While we thus seen that the path integral formulations of the second and fourth order oscillator theories mirror each other in quite a few ways, in one particular regard however there is a quite substantive difference between them, namely in the normalizability of the energy eigenstates. While the positive energy eigenstates of the second order oscillator theory are fully normalizable using a conventional $\int d q \psi^{*}(q) \psi(q)$ norm, to determine whether or not this is also the case in the fourth order theory, we need to determine an appropriate norm for the eigenstates of the wave equation of Eq. (B2). To this end we note that for any two of its eigenstates, manipulation of the wave equation yields the kinematic identity

$$
\begin{aligned}
\left(E_{1}-E_{2}\right) \psi_{2}^{*}(q, x) \psi_{1}(q, x)= & -\frac{1}{2 \gamma} \frac{\partial}{\partial x}\left[\psi_{2}^{*}(q, x) \frac{\partial \psi_{1}(q, x)}{\partial x}-\frac{\partial \psi_{2}^{*}(q, x)}{\partial x} \psi_{1}(q, x)\right] \\
& -i x \frac{\partial}{\partial q}\left[\psi_{2}^{*}(q, x) \psi_{1}(q, x)\right] .
\end{aligned}
$$

Consequently, if we define a norm of the form

$$
N_{12}=\int_{-\infty}^{\infty} d q \int_{-\infty}^{\infty} d x \psi_{2}^{*}(q, x) \psi_{1}(q, x)
$$

for it we will find that states which are sufficiently bounded as $q \rightarrow \pm \infty$ and as $x \rightarrow \pm \infty$ will be orthogonal, with Eq. (B13) thus furnishing us with the appropriate norm for the orthonormality condition $N_{m n}=\delta_{m n}$. However, on applying this norm to the eigenfunctions of Eq. (B2), not only do we find that the negative energy sector modes are non-normalizable, additionally we find that the positive energy ones are non-normalizable too, since both of the norms

$$
\begin{aligned}
& N\left(\psi_{0}\right)=\int_{-\infty}^{\infty} d q \int_{-\infty}^{\infty} d x \exp \left[\gamma \omega_{1} \omega_{2}\left(\omega_{1}+\omega_{2}\right) q^{2}-\gamma\left(\omega_{1}+\omega_{2}\right) x^{2}\right] \\
& N\left(\hat{\psi}_{0}\right)=\int_{-\infty}^{\infty} d q \int_{-\infty}^{\infty} d x \exp \left[-\gamma \omega_{1} \omega_{2}\left(\omega_{1}+\omega_{2}\right) q^{2}+\gamma\left(\omega_{1}+\omega_{2}\right) x^{2}\right]
\end{aligned}
$$

will diverge no matter what choice of sign we take for $\gamma\left(\omega_{1}\right.$ and $\omega_{2}$ are both taken to be positive). Thus unlike the standard one-dimensional harmonic oscillator case where a normalizability criterion does distinguish between the positive and negative energy eigenstates (the diverging $e^{m \omega q^{2} / 2 \hbar}$ based modes not being normalizable), in the fourth order oscillator case not only are the first-quantized negative energy eigenstates not normalizable, the first-quantized positive energy eigenstates are not normalizable either. 
Nonetheless, despite this, the path integral which is associated with these nonnormalizable first-quantized positive energy eigenstates is still well-behaved with no sign of any divergence in Eq. (B9), and with the contributions of the various eigenstates in the expansion of Eq. (B10) appearing with finite coefficients. Now as such, the overlap integral $\langle(q(T), \dot{q}(T)) \mid(q(0), \dot{q}(0))\rangle$ represents the probability that a state which is localized at $t=0$ will evolve into one which is localized at $t=T$. As such, localized states have to be constructed out of linear superpositions of the modes of a complete basis, with the positive energy sector solutions to the Schrödinger equation of Eq. (B2) thus being seen to be complete. The fact that eigenmodes might not be normalizable does not prevent them from being complete (though orthonormal basis modes are of course complete). Indeed, while plane waves for instance might not be normalizable, one can still construct localized packets out of them which are. Moreover, this is in fact a quite general phenomenon, with it being explicitly shown in [25, 26] that via destructive interference outside the steps, it is possible to construct localized square steps even when basis modes are highly divergent. Orthonormality is thus sufficient for completeness but not at all necessary. For the Pais-Uhlenbeck oscillator then, we see that from the behavior of $\langle(q(T), \dot{q}(T)) \mid(q(0), \dot{q}(0))\rangle$ at asymptotic Euclidean time, we can identify which modes are contained in the initial localized packet at $t=0$ and into which modes they evolve at the final $t=T$. For the unequal frequency case we thus see that completeness requires both the positive signature and the negative signature families of positive energy eigenmodes (but needs no negative energy modes), and that these modes do not mix with each other under temporal evolution. However, for the equal frequency case, we see from Eq. (B11) that its single family of positive energy modes is complete all on its own, with no basis states thus being missed, and with negative signatured states not then being needed for completeness at all.

Now while we have encountered non-normalizable modes in the first-quantized formulation of the fourth order theory, as we recall, in its second-quantized formulation, no infinite norm energy eigenstates are encountered at all. And even though there are negative norm states in the second-quantized unequal frequency theory, as we have seen, in the equal frequency limit no such states appear in the eigenspectrum of the Hamiltonian, with there being no on-shell states of negative energy or negative norm at all. The second quantization of the equal frequency Pais-Uhlenbeck oscillator theory as based on the Dirac constraint Hamiltonian of Eq. (22) thus leads us directly to a fully acceptable physical theory. 


\section{APPENDIX C: HIDDEN SYMMETRY OF THE FOURTH ORDER THEORY}

To provide additional insight into the structure of the eigenspectrum of $H(\epsilon=0)$ of Eq. (55), we conclude this paper by showing that in the equal frequency limit, the fourth order theory acquires a hidden symmetry which sharply constrains the energy eigenspectrum. To orient the discussion, we recall that for a one-dimensional simple harmonic oscillator, both the Hamiltonian $H=\hbar \omega\left(a^{\dagger} a+1 / 2\right)$ and the commutation relation $\left[a, a^{\dagger}\right]=1$ are left invariant under the transformation $a \rightarrow e^{i \theta} a$. Such a transformation is without physical content as the phase change can be absorbed in a redefinition of the phase of the vacuum $|\Omega\rangle$. Moreover, this remains true even for the two-oscillator realization of the unequal frequency Pais-Uhlenbeck theory as given in Eqs. (41) and (40) where both the Hamiltonian and the commutation relations are left invariant under $a_{1} \rightarrow e^{i \theta} a_{1}, a_{2} \rightarrow e^{i \phi} a_{2}$, since in the absence of any $a_{1}, a_{2}$ sector cross-terms, both phases can be absorbed in a redefinition of the vacuum which both $a_{1}$ and $a_{2}$ annihilate.

If we now transcribe to the $a, b$ basis given in Eq. (44), we will find that under these phase transformations the $a$ and $b$ operators will transform as

$$
\begin{aligned}
& a \rightarrow \frac{1}{2}\left[e^{i \theta}\left(1+\frac{\epsilon}{2 \omega}\right)+e^{i \phi}\left(1-\frac{\epsilon}{2 \omega}\right)\right] a+\frac{1}{2}\left[\left(e^{i \theta}-e^{i \phi}\right)\left(\frac{2 \omega}{\epsilon}-\frac{\epsilon}{2 \omega}\right)\right] b \\
& b \rightarrow \frac{1}{2}\left[e^{i \theta}\left(1-\frac{\epsilon}{2 \omega}\right)+e^{i \phi}\left(1+\frac{\epsilon}{2 \omega}\right)\right] b+\frac{\epsilon}{4 \omega}\left(e^{i \theta}-e^{i \phi}\right) a
\end{aligned}
$$

As well as the trivial symmetry associated with setting $\theta=\phi\left(\right.$ viz. $\left.a \rightarrow e^{i \theta} a, b \rightarrow e^{i \theta} b\right)$, to obtain a non-trivial symmetry as we let $\epsilon$ go to zero we define

$$
\theta=\frac{\epsilon \psi}{2 \omega}, \phi=-\frac{\epsilon \psi}{2 \omega}
$$

and for zero $\epsilon$ thus obtain

$$
a \rightarrow a+i \psi b \quad, \quad b \rightarrow b \quad, \quad a^{\dagger} \rightarrow a^{\dagger}-i \psi b^{\dagger} \quad, \quad b^{\dagger} \rightarrow b^{\dagger}
$$

with the $b$ sector being found to transform into itself, but not $a$. For this transformation we note also that

$$
a^{\dagger} b+b^{\dagger} a \rightarrow\left(a^{\dagger}-i \psi b^{\dagger}\right) b+b^{\dagger}(a+i \psi b)=a^{\dagger} b+b^{\dagger} a
$$

Consequently, the transformation of Eq. (C3) with arbitrary $\psi$ leaves the equal frequency Hamiltonian $H(\epsilon=0) \sim a^{\dagger} b+b^{\dagger} a+2 b^{\dagger} b$ of Eq. (155) invariant. Moreover, with the equal 
frequency commutation relations of Eq. (46) taking the form

$$
\left[a, a^{\dagger}\right]=0 \quad, \quad\left[a, b^{\dagger}\right]=\frac{1}{8 \gamma \omega^{3}} \quad, \quad\left[b, a^{\dagger}\right]=\frac{1}{8 \gamma \omega^{3}} \quad, \quad\left[b, b^{\dagger}\right]=0 \quad, \quad[a, b]=0,
$$

we see that the commutation relations are left invariant too.

We thus recognize the transformation of Eq. (C3) as a continuous symmetry of the equal frequency theory. Moreover, unlike the unequal frequency case, this particular symmetry is not without content. Specifically, since this symmetry leaves the Hamiltonian invariant, the energy eigenstates must be irreducible under it. Consequently, only the $b^{\dagger}$ sector modes can be energy eigenstates, and not the $a^{\dagger}$ based ones. This hidden symmetry thus prevents the $a^{\dagger}$ sector modes from being energy eigenstates, to thereby leave the $H(\epsilon=0)$ Hamiltonian in the defective form we identified earlier. Moreover, with only the $b^{\dagger}$ sector modes being invariant under the symmetry, these are then the only eigenmodes of $H(\epsilon=0)$, with no new eigenmodes being able to emerge to replace the $a^{\dagger}$ sector modes which $H(\epsilon)$ loses in the $\epsilon \rightarrow 0$ limit. The enumeration of the $H(\epsilon=0)$ eigenspectrum is thus complete, with it having the same dimensionality as that of a one-dimensional oscillator, even while the dimensionality of the unequal frequency energy spectrum is that of a two-dimensional one.

In the $(a, b)$ space the transformation of Eq. (C3) can be represented as the $2 \times 2$ matrix

$$
M^{-1}=\left(\begin{array}{cc}
1 & i \psi \\
0 & 1
\end{array}\right) \text {. }
$$

With its inverse and the adjoint of its inverse being given by

$$
M=\left(\begin{array}{cc}
1 & -i \psi \\
0 & 1
\end{array}\right), M^{\dagger}=\left(\begin{array}{cc}
1 & 0 \\
i \psi & 1
\end{array}\right)
$$

the identity

$$
\left(\begin{array}{ll}
a & b
\end{array}\right)\left(\begin{array}{ll}
0 & 1 \\
1 & 2
\end{array}\right)\left(\begin{array}{l}
a \\
b
\end{array}\right)=\left(\begin{array}{ll}
a & b
\end{array}\right)\left(M^{-1}\right)^{\dagger} M^{\dagger}\left(\begin{array}{ll}
0 & 1 \\
1 & 2
\end{array}\right) M M^{-1}\left(\begin{array}{l}
a \\
b
\end{array}\right)
$$

then requires that Hamiltonian of Eq. (155) transform as $H \rightarrow M^{\dagger} H M$. The actual invariance of the $H(\epsilon=0)$ under the transformation of Eq. (C3) thus requires that it obey

$$
M^{\dagger}\left(\begin{array}{ll}
0 & 1 \\
1 & 2
\end{array}\right) M=\left(\begin{array}{cc}
1 & 0 \\
i \psi & 1
\end{array}\right)\left(\begin{array}{cc}
0 & 1 \\
1 & 2
\end{array}\right)\left(\begin{array}{cc}
1 & -i \psi \\
0 & 1
\end{array}\right)=\left(\begin{array}{ll}
0 & 1 \\
1 & 2
\end{array}\right)
$$

and one can check immediately that this relation does indeed hold. Now we note that $M^{\dagger}$ is not equal to $M^{-1}$, with the transformation matrix $M$ thus not being unitary. The 
symmetry associated with $M$ is thus not a normal unitary symmetry. Now ordinarily one restricts to unitary transformations since one is usually working in a Hilbert space with a positive definite metric and positive norm states and one wants to conserve probabilities. However, in the equal frequency Pais-Uhlenbeck theory the $b^{\dagger}|\Omega\rangle$ and $a^{\dagger}|\Omega\rangle$ states have zero norm and finite overlaps, viz.

$$
\left\langle\Omega\left|a a^{\dagger}\right| \Omega\right\rangle=0 \quad, \quad\left\langle\Omega\left|b b^{\dagger}\right| \Omega\right\rangle=0 \quad, \quad\left\langle\Omega\left|a b^{\dagger}\right| \Omega\right\rangle=\frac{1}{8 \gamma \omega^{3}} \quad, \quad\left\langle\Omega\left|b a^{\dagger}\right| \Omega\right\rangle=\frac{1}{8 \gamma \omega^{3}},
$$

to thus allow us to consider more general symmetries. And in fact under the transformations of Eq. (C3), use of the relations of Eq. (C10) shows that overlaps transform as

$$
\begin{aligned}
& \left\langle\Omega\left|a a^{\dagger}\right| \Omega\right\rangle \rightarrow\left\langle\Omega\left|(a+i \psi b)\left(a^{\dagger}-i \psi b^{\dagger}\right)\right| \Omega\right\rangle=\left\langle\Omega\left|a a^{\dagger}\right| \Omega\right\rangle, \\
& \left\langle\Omega\left|b b^{\dagger}\right| \Omega\right\rangle \rightarrow\left\langle\Omega\left|b b^{\dagger}\right| \Omega\right\rangle, \\
& \left\langle\Omega\left|a b^{\dagger}\right| \Omega\right\rangle \rightarrow\left\langle\Omega\left|(a+i \psi b) b^{\dagger}\right| \Omega\right\rangle=\left\langle\Omega\left|a b^{\dagger}\right| \Omega\right\rangle, \\
& \left\langle\Omega\left|b a^{\dagger}\right| \Omega\right\rangle \rightarrow\left\langle\Omega\left|b\left(a^{\dagger}-i \psi\right) b^{\dagger}\right| \Omega\right\rangle=\left\langle\Omega\left|b a^{\dagger}\right| \Omega\right\rangle,
\end{aligned}
$$

and are thus actually invariant even though $M$ is not unitary.

[1] K. S. Stelle, Phys. Rev. D 16, 953 (1977); Gen. Relativ. Gravit. 9, 353 (1978).

[2] P. D. Mannheim and A. Davidson, "Fourth order theories without ghosts", hep-th/0001115, January 2000.

[3] S. W. Hawking and T. Hertog, Phys. Rev. D 65, 103515 (2002).

[4] P. D. Mannheim and A. Davidson, Phys. Rev. A 71, 042110 (2005).

[5] P. A. M. Dirac, "Lectures on Quantum Mechanics", Belfer Graduate School of Science, Yeshiva University Press, New York (1964).

[6] A. Pais and G. E. Uhlenbeck, Phys. Rev. 79, 145 (1950).

[7] The work described here is based on the use of a Dirac constraint quantization of the prototype second plus fourth order Pais-Uhlenbeck theory as given in 2, 4]. An alternate method for handling the constraints of the Pais-Uhlenbeck theory, one based on a Feynman path integral quantization, was introduced in [3], and further discussion of the ghost structure of the theory may be found in A. V. Smilga, Nucl. Phys. B 706, 598 (2005); Phys. Lett. B 632, 433 (2006); V. O. Rivelles, Phys. Lett. B 577, 137 (2003); and O. Sarioglu and B. Tekin, "Topologically massive gravity as a Pais-Uhlenbeck oscillator", gr-qc/0608085. 
[8] By use of the relations $\left(\partial_{t}^{2}-\nabla^{2}\right)[\theta(t-r) / 8 \pi]=\delta(t-r) / 4 \pi r,\left(\partial_{t}^{2}-\nabla^{2}\right)[\delta(t-r) / 4 \pi r]=\delta^{4}(x)$, we can check directly that the propagators $D^{(4)}(x, M=0 ; \mathrm{RET})$ and $D^{(4)}(x, M=0 ; \mathrm{ADV})$ as given in Eq. ([6) do indeed obey $\left(-\partial_{t}^{2}+\nabla^{2}\right)^{2} D^{(4)}(x, M=0)=\delta^{4}(x)$. In addition, we could also construct the retarded $D^{(4)}(x, M=0 ; \mathrm{RET})=1 /(2 \pi)^{4} \int d^{4} k e^{i k \cdot x} /\left(k^{2}-i \epsilon \epsilon\left(k^{0}\right)\right)^{2}$ as the $M^{2} \rightarrow$ 0 limit of $-\left(\partial / \partial M^{2}\right)\left[1 /(2 \pi)^{4} \int d^{4} k e^{i k \cdot x} /\left(k^{2}+M^{2}-i \epsilon \epsilon\left(k^{0}\right)\right)\right]=\left(\partial / \partial M^{2}\right) D^{(2)}(x, M ; \mathrm{RET})$, and likewise for $D^{(4)}(x, M=0$; $\mathrm{ADV})$. Moreover, from the explicit form given for $D^{(4)}(x, M=$ $0 ; \mathrm{RET})$ as $\theta(t-r) / 8 \pi$, we note that whatever one might think may possibly be wrong with the fourth order theory, even with possible ghosts, causality of the theory is not in question since the retarded $D^{(4)}(x, M=0 ; \mathrm{RET})$ does not take support outside the $t^{2}=|\bar{x}|^{2}=r^{2}$ light cone. [In passing we should note that unlike the retarded second order massless propagator $D^{(2)}(x, M=0)$ which only takes support on the 4-dimensional light cone, the retarded fourth order massless propagator $D^{(4)}(x, M=0)$ also takes support within it. Moreover, this is not actually uncommon for massless propagators, with the retarded second order massless propagator in two dimensions for instance being given by $\theta(t-|x|) / 2=\theta(t) \theta[(t-|x|)(t+$ $|x|)] / 2=\theta(t) \theta\left(t^{2}-x^{2}\right) / 2$. (Use of $\partial_{x}|x|=\theta(x)-\theta(-x), \partial_{x}^{2}|x|=2 \delta(x)$ shows that $\left(\partial_{t}^{2}-\partial_{x}^{2}\right) \theta(t-$ $|x|) / 2=\delta(t) \delta(x)$.) In fact since theta functions are dimensionless while delta functions are not $\left(\delta(t-|\bar{x}|) / 2|\bar{x}|=\theta(t) \delta\left(t^{2}-\bar{x}^{2}\right)\right.$ has dimension $\left.x^{-2}\right)$, matching the $x^{2 n-D}$ dimension of the general $\int d^{D} p e^{i p \cdot x} /\left(p^{2}\right)^{n}$ might not be achievable by involving delta functions, in which case Lorentz invariance and causality would necessitate involving theta functions instead.]

[9] In the terminology of the method of Dirac constraints, both of these particular types of constraints are said to be primary.

[10] M. Ostrogradski, "Memoires sur les equations differentielles relatives au probleme des isoperimetres", Mem. Ac. St. Petersbourg VI 4, 385 (1850).

[11] In the language of Feynman path integral quantization, the path integral measure consists of precisely the same set of phase space paths over which the stationary classical Hamilton variation is made, an issue we discuss in more detail in appendix A.

[12] The linear in time growth of the temporal runaway is not seen to lead to any runaway energy since it is the Ostrogradski Hamiltonian of Eq. (28) which is the correct Hamiltonian for the problem, with its explicit $\ddot{q}$ dependent terms serving to maintain the time independence of the energy. Temporal runaways are thus not seen to be the problem for fourth order theories that they are often thought to be. 
[13] D. G. Boulware, G. T. Horowitz, and A. Strominger, Phys. Rev. Lett. 50, 1726 (1983).

[14] P. D. Mannheim and D. Kazanas, Astrophys. J. 342, 635 (1989).

[15] In P. D. Mannheim and D. Kazanas, Gen. Relativ. Gravit. 26, 337 (1994) it is shown that just like the solutions to the Pais-Uhlenbeck theory, the conformal gravity solution of Eqs. (36) and (37) is also a solution to a fourth box operator equation, viz. the equation $\nabla^{4} B(r)=0$ which the static, spherically symmetric conformal gravity vacuum solution is required to obey.

[16] In and of itself having on-shell energy eigenstates of negative norm does not actually doom the theory. Rather, it would be having S-matrix transitions which connect these negative norm states with the positive norm ones which would actually render the theory unphysical. It thus of interest to note that via their path-integral quantization procedure Hawking and Hertog $[\underline{3}]$ have found that in the presence of interactions such transitions do not in fact occur.

[17] Once we have two zero norm states, then from them we can construct two other states one with positive norm and the other with negative norm, viz. $\left(a^{\dagger} \pm b^{\dagger}\right)|\Omega\rangle$ which according to Eq. (46) have norms $\pm 2 \mu(\epsilon=0)= \pm 1 / 4 \gamma w^{3}$. However, since neither of these states is an energy eigenstate, both only exist off shell. It is thus the fact that the zero norm state $a^{\dagger}|\Omega\rangle$ is not an eigenstate of the $H(\epsilon=0)$ Hamiltonian which prevents $H(\epsilon=0)$ from having any on-shell states of negative norm.

[18] By exploiting a hidden symmetry that the Pais-Uhlenbeck theory acquires in the $\epsilon \rightarrow 0$ limit, we show in appendix $\mathrm{C}$ that there are no compensating Fock space states elsewhere in the theory that suddenly become energy eigenstates in the $\epsilon \rightarrow 0$ limit.

[19] While the would-be ghosts cannot go on shell, they are still present in the full off-shell Fock space of the equal frequency Pais-Uhlenbeck theory as the Fock space still has the dimensionality of a two-dimensional oscillator. That these ghost states are in fact able to appear off shell without then needing to have to appear on shell as well is because the underlying quantum-mechanical Hilbert space is not positive definite, with the usual Landau-Cutkowsky cutting rules not then applying, with off-shell intermediate states in Feynman diagrams not then being obligated to have to materialize as on-shell states as well. Since the ghost states can contribute off shell, just like the equally ghost-signatured Pauli-Villars regulators, they can serve to regulate Feynman diagrams far off the mass shell and produce better convergence than would be obtained in their absence. Because of its off-shell ghost graviton then, the fourth order conformal invariant gravitational theory with action $I_{W}=-\alpha_{g} \int d^{4} x(-g)^{1 / 2} C_{\lambda \mu \nu \kappa} C^{\lambda \mu \nu \kappa}$ 
where $C_{\lambda \mu \nu \kappa}$ is the Weyl tensor and the coupling constant $\alpha_{g}$ is dimensionless has far better convergence properties in the ultraviolet than the standard second order Einstein theory (with action $\left.I_{E H}=-(1 / 16 \pi G) \int d^{4} x(-g)^{1 / 2} R_{\alpha}^{\alpha}\right)$, with the ghost-signatured graviton which is to now accompany the regular positive-signatured graviton off shell serving to cancel the leading ultraviolet divergences and make the conformal theory power counting renormalizable. Macroscopically, conformal gravity is a viable alternate gravitational theory which naturally resolves [P. D. Mannheim, Prog. Part. Nucl. Phys. 56, 340 (2006)] the dark matter, dark energy and cosmological constant problems which currently afflict the standard Einstein theory. An extension of the structure found for the equal frequency Pais-Uhlenbeck theory to conformal gravity would then leave it free of on-shell ghosts and render it microscopically viable as well.

[20] C. M. Bender, D. C. Brody and H. F. Jones, Am. J. Phys. 71, 1095 (2003).

[21] In the unequal frequency theory the introduction of a $C$ operator in the $a_{1}^{\dagger}|\Omega\rangle, a_{2}^{\dagger}|\Omega\rangle$ space which acts as the Pauli matrix $\sigma_{3}$ allows us to define one-particle sector matrix elements of the form $\left\langle\Omega\left|a_{1} C a_{1}^{\dagger}\right| \Omega\right\rangle=1 /\left[2 \gamma \omega_{1}\left(\omega_{1}^{2}-\omega_{2}^{2}\right)\right],\left\langle\Omega\left|a_{1} C a_{2}^{\dagger}\right| \Omega\right\rangle=0,\left\langle\Omega\left|a_{2} C a_{1}^{\dagger}\right| \Omega\right\rangle=0,\left\langle\Omega\left|a_{2} C a_{2}^{\dagger}\right| \Omega\right\rangle=$ $1 /\left[2 \gamma \omega_{2}\left(\omega_{1}^{2}-\omega_{2}^{2}\right)\right]$. The fact that the $a_{1}^{\dagger}|\Omega\rangle, a_{2}^{\dagger}|\Omega\rangle$ sector can be recast in an orthonormalizable form with no negative norm states buttresses the finding of [3] that the unequal frequency Pais-Uhlenbeck theory is viable.

[22] We use the term "effective" here since even though the original classical Pais-Uhlenbeck theory was only defined in a one-dimensional coordinate space, as we see, its quantum version is to be associated with a two-dimensional one, with the two quantum operators $q$ and $x$ having eigenvalues which collectively span a two-dimensional classical coordinate space. Amusingly, in this sense then, higher derivative theories are able to generate additional spacetime dimensions.

[23] We should stress that using the Schrödinger equation to fix the normalization of the path integral already requires knowing the quantum-mechanical Hamiltonian, and thus requires that the Dirac constraint quantization procedure had already been carried out.

[24] For the one-dimensional harmonic oscillator, the analogous factor which is to normalize $\langle q(T) \mid q(0)\rangle$ in Eq. (A20) is given as $1 /(\sin \omega T)^{1 / 2}$, with its Euclidean continuation asymptoting to $e^{-\omega \tau / 2}$, to precisely yield the ground state zero point energy.

[25] P. D. Mannheim, "Brane-Localized Gravity", World Scientific Press, New Jersey (2005).

[26] P. D. Mannheim and I. Simbotin, "Completeness of non-normalizable modes", hep-th/0607090. 